\title{
DISCRETE FOURIER ANALYSIS WITH LATTICES ON PLANAR DOMAINS
}

\author{
HUIYUAN LI, JIACHANG SUN, AND YUAN XU
}

\begin{abstract}
A discrete Fourier analysis associated with translation lattices is developed recently by the authors. It permits two lattices, one determining the integral domain and the other determining the family of exponential functions. Possible choices of lattices are discussed in the case of lattices that tile $\mathbb{R}^{2}$ and several new results on cubature and interpolation by trigonometric, as well as algebraic, polynomials are obtained.
\end{abstract}

\section{INTRODUCTION}

A framework of discrete Fourier analysis associated with translation tiling was developed recently in [7, based on the principle that if $\Omega$ is a bounded open set that tiles $\mathbb{R}^{d}$ with the lattice $L=A \mathbb{Z}^{d}$, then the family of exponentials $\left\{\mathrm{e}^{2 \pi i \alpha \cdot x}\right.$ : $\left.\alpha \in L^{\perp}\right\}$, where $L^{\perp}=A^{-\operatorname{tr}} \mathbb{Z}^{d}$ is the dual lattice of $L$, forms an orthonormal basis in $L^{2}(\Omega)(4)$. Our set up permits two lattices, one determining the integral domain and the other determining the exponentials that are orthogonal under the discrete inner product. The case that both lattices have the regular hexagon as fundamental domain was studied in [7] to illustrate the main set up, which leads to new cubature formula and Lagrange interpolation for trigonometric polynomials on hexagonal domains and equilateral triangles, as well as results for algebraic polynomials on the region bounded by Steiner's hypocycloid. This is extended to three dimension in $[9$, giving results on cubature and interpolation on the rhombic dodecahedron and the tetrahedron, and further extended to $\mathbb{R}^{d}$ in $\left[10\right.$ for $\mathcal{A}_{d}$ type lattice. In 8 , the two lattices are chosen differently with fundamental domains being a square and a rhombic (rotation of the square by $90^{\circ}$ ), respectively. The choice leads to, surprisingly, one family of minimal cubature for product Chebyshev weight on $[-1,1]^{2}$, first discovered by working with common zeros of orthogonal polynomials of two variables. An extension to three dimension gives a family of cubature formulas on the cube that have the smallest number of nodes among all known formulas, which coincides, rather surprisingly, with the cubature discovered in [1] by a totally different method.

The two lattices in 8 , were chosen for the purpose of obtaining algebraic cubature formulas on the square. Its success prompts us to ask what other choices are possible. In the present paper, we try to answer this question in the case of $\mathbb{R}^{2}$. Up to affine transforms, there are essentially two types of translation tiling

Date: June 4, 2018.

1991 Mathematics Subject Classification. 41A05, 41A10.

Key words and phrases. Discrete Fourier series, trigonometric, lattices, cubature, interpolation.

The first authors was supported by NSFC Grants 10601056 and 10971212. The second author was supported by NSFC Grant 60970089 and National Basic Research Program Grant 2005CB321702. 
in $\mathbb{R}^{2}$ with fundamental domain being either squares or regular hexagons. Their combinations in our discrete Fourier analysis, however, yield several distinct cases, including several cases not covered in our previous studies. One that is of particular interesting has one tiling sets as the regular hexagon and the other as the rotation of the regular hexagon by $90^{\circ}$ (see Section 3.5), which leads to another set of cubature and interpolation on the equilateral triangle, different from those obtained in 7. In order to present the main idea without being overwhelmed by notations and numerous formulas, we shall work mostly with cubature formulas, a central part but by no means all of discrete Fourier analysis, unless other results are deemed novel enough to warrant inclusion.

The paper is organized as follows. In the following section we recall the framework developed in [7] and use it to treat the classical product discrete Fourier analysis on the plane, which illustrates well what can be expected in the non-classical settings. Section 3 is divided into a number of subsections, each deals with one distinct choice of two lattices.

\section{Discrete Fourier analysis With Lattice}

In the first subsection, we give a succinct recount of the framework of discrete Fourier analysis with tiling in 7 . We refer to 1 for lattices, tiling and various related topics, and refer to 2, 14 for some applications of discrete Fourier analysis in several variables. In the second subsection, we illustrate the general theory by using it to recover the classical product discrete Fourier analysis on the square.

2.1. Discrete Fourier analysis. A lattice $L$ in $\mathbb{R}^{d}$ is a discrete subgroup $L=$ $L_{A}:=A \mathbb{Z}^{d}$, where $A$, called a generator matrix, is nonsingular. A bounded set $\Omega$ of $\mathbb{R}^{d}$, called the fundamental domain of $L$, is said to tile $\mathbb{R}^{d}$ with the lattice $L$ if

$$
\sum_{\alpha \in L} \chi_{\Omega}(x+\alpha)=1, \quad \text { for almost all } x \in \mathbb{R}^{d},
$$

where $\chi_{\Omega}$ denotes the characteristic function of $\Omega$. We write this as $\Omega+L=\mathbb{R}^{d}$. For a given lattice $L_{A}$, the dual lattice $L_{A}^{\perp}$ is given by $L_{A}^{\perp}=A^{-\operatorname{tr}} \mathbb{Z}^{d}$. According to a result of Fuglede [4, a bounded open set $\Omega$ tiles $\mathbb{R}^{d}$ with the lattice $L$ if, and only, $\left\{\mathrm{e}^{2 \pi i \alpha \cdot x}: \alpha \in L^{\perp}\right\}$ is an orthonormal basis with respect to the inner product

$$
\langle f, g\rangle_{\Omega}=\frac{1}{\operatorname{mes}(\Omega)} \int_{\Omega} f(x) \overline{g(x)} d x .
$$

For $L=A \mathbb{Z}^{d}$, the measure of $\Omega$ is equal to $|\operatorname{det}(A)|$. Since $L_{A}^{\perp}=A^{-\operatorname{tr}} \mathbb{Z}^{d}$, we can write $\alpha=A^{-\operatorname{tr}} k$ for $\alpha \in L_{A}^{\perp}$ and $k \in \mathbb{Z}^{d}$, so that $\mathrm{e}^{2 \pi i \alpha \cdot x}=\mathrm{e}^{2 \pi i k^{\text {tr }} A^{-1} x}$.

For our discrete Fourier analysis, the boundary of $\Omega$ matters. We shall fix an $\Omega$ such that $0 \in \Omega$ and $\Omega+A \mathbb{Z}^{d}=\mathbb{R}^{d}$ holds pointwisely and without overlapping.

Definition 2.1. Let $\Omega_{A}$ and $\Omega_{B}$ be the fundamental domains of $A \mathbb{Z}^{d}$ and $B \mathbb{Z}^{d}$, respectively. Assume all entries of the matrix $N:=B^{\operatorname{tr}} A$ are integers. Define

$$
\Lambda_{N}:=\left\{k \in \mathbb{Z}^{d}: B^{- \text {tr }} k \in \Omega_{A}\right\} \quad \text { and } \quad \Lambda_{N}^{\dagger}:=\left\{k \in \mathbb{Z}^{d}: A^{- \text {tr }} k \in \Omega_{B}\right\} .
$$

Furthermore, define the finite dimensional subspace of exponential functions

$$
\mathcal{H}_{N}:=\operatorname{span}\left\{\mathrm{e}^{2 \pi i k^{\mathrm{tr}} A^{-1} x}, k \in \Lambda_{N}^{\dagger}\right\} .
$$

The main result in the discrete Fourier analysis is the following theorem: 
Theorem 2.2. Let $A, B$ and $N$ be as in Definition 2.1. Define

$$
\langle f, g\rangle_{N}=\frac{1}{|\operatorname{det}(N)|} \sum_{j \in \Lambda_{N}} f\left(B^{-\operatorname{tr}} j\right) \overline{g\left(B^{-\operatorname{tr}} j\right)}
$$

for $f, g$ in $C\left(\Omega_{A}\right)$, the space of continuous functions on $\Omega_{A}$. Then

$$
\langle f, g\rangle_{\Omega_{A}}=\langle f, g\rangle_{N}, \quad f, g \in \mathcal{H}_{N} .
$$

It follows readily that (2.2) gives a cubature formula exact for functions in $\mathcal{H}_{N}$. Furthermore, it also implies a Lagrange interpolation by exponential functions. Let $\mathcal{I}_{N} f$ denote the Fourier expansion of $f$ in $\mathcal{H}_{N}$ with respect to the inner product $\langle\cdot, \cdot\rangle_{N}$, which can be expressed as

$$
\mathcal{I}_{N} f(x)=\sum_{k \in \Lambda_{N}} f\left(B^{-\operatorname{tr}} k\right) \Psi_{\Omega_{B}}^{A}\left(x-B^{-\operatorname{tr}} k\right), \quad f \in C\left(\Omega_{A}\right),
$$

where

$$
\Psi_{\Omega_{B}}^{A}(x)=\frac{1}{|\operatorname{det}(N)|} \sum_{j \in \Lambda_{N}^{\dagger}} \mathrm{e}^{2 \pi i j^{\mathrm{tr}} A^{-1} x} .
$$

Theorem 2.3. Let $A, B$ and $N$ be as in Definition 2.1. Then $\mathcal{I}_{N}$ is the unique interpolation operator on $N$ in $\mathcal{H}_{N}$; that is,

$$
\mathcal{I}_{N} f\left(B^{-\operatorname{tr}} j\right)=f\left(B^{-\operatorname{tr}} j\right), \quad \forall j \in \Lambda_{N} .
$$

In particular, $\# \Lambda_{N}=\# \Lambda_{N^{\mathrm{tr}}}=|\operatorname{det}(N)|$. The cubature formula and the Lagrange interpolation are for functions that are periodic with respect to the lattice $A \mathbb{Z}^{d}$, which are functions satisfying

$$
f(x+A k)=f(x) \quad \text { for all } k \in \mathbb{Z}^{d} .
$$

The function $x \mapsto \mathrm{e}^{2 \pi i k^{\mathrm{tr}} A^{-1} x}$ is periodic with respect to the lattice $A \mathbb{Z}^{d}$.

2.2. Classical discrete Fourier analysis. We deduce the classical result on the plane (cf. 2, 23]) from the general theory described above. As mentioned in the introduction, we shall limit our consideration to cubature formulas. The result hints at what is possible in the non-classical cases in the rest of the paper.

For $n \in \mathbb{N}$, let $A=I$, the identity matrix, and $B=2 n I$. Then $N=B^{\operatorname{tr}} A=2 n I$ has all integer entries. Let $\Omega_{A}=\left[-\frac{1}{2}, \frac{1}{2}\right)^{2}$, which tiles $\mathbb{R}^{2}$ with $\mathbb{Z}^{2}$ pointwisely and without overlapping. We shall write $\Lambda_{n}, \Lambda_{n}^{\dagger}, \mathcal{H}_{n}$ in place of $\Lambda_{N}, \Lambda_{N}^{\dagger}, \mathcal{H}_{N}$. Then

$$
\Lambda_{n}=\Lambda_{n}^{\dagger}=\left\{k \in \mathbb{Z}^{2}: k \in[-n, n)^{2}\right\} \quad \text { and } \quad \mathcal{H}_{n}=\operatorname{span}\left\{\mathrm{e}^{2 \pi i k \cdot x}: k \in \Lambda_{n}^{\dagger}\right\} .
$$

It is clear that $\# \Lambda_{n}=(2 n)^{2}$. The equation (2.2) in this setting becomes

$$
\int_{\left[-\frac{1}{2}, \frac{1}{2}\right]^{2}} f(x) \overline{g(x)} d x=\frac{1}{4 n^{2}} \sum_{k_{1}=-n}^{n-1} \sum_{k_{2}=-n}^{n-1} f\left(\frac{k_{1}}{2 n}, \frac{k_{2}}{2 n}\right) \overline{g\left(\frac{k_{1}}{2 n}, \frac{k_{2}}{2 n}\right)}, \quad f, g \in \mathcal{H}_{n} .
$$

To illustrate what can be done on cubature, we state the results in stages.

Stage 1. It is easy to see that (2.5) yields a cubature formula

$$
\int_{\left[-\frac{1}{2}, \frac{1}{2}\right]^{2}} f(x) d x=\frac{1}{4 n^{2}} \sum_{k_{1}=-n}^{n-1} \sum_{k_{2}=-n}^{n-1} f\left(\frac{k_{1}}{2 n}, \frac{k_{2}}{2 n}\right), \quad \forall f \in \mathcal{H}_{2 n-1}^{*},
$$


where $\mathcal{H}_{n}^{*}:=\operatorname{span}\left\{\mathrm{e}^{2 \pi i k \cdot x}: k \in[-n, n]^{2} \cap \mathbb{Z}^{2}\right\}$. The set of nodes of cubature (2.6) is not symmetric on $\left[-\frac{1}{2}, \frac{1}{2}\right]^{2}$, since it has points on only part of the boundary of the square. We would like to have cubature whose nodes is symmetric on the square.

Stage 2. We construct cubature formulas for $\mathcal{H}_{2 n-1}^{*}$ that have symmetric nodes on the square. Such a cubature is invariant under sign changes in both variables. We can in fact obtain two such formulas from (2.6). The first one is obtained upon using the periodicity of the functions in the sums in the right hand side,

$$
\int_{\left[-\frac{1}{2}, \frac{1}{2}\right]^{2}} f(x) d x=\frac{1}{4 n^{2}} \sum_{k_{1}=-n}^{n} \sum_{k_{2}=-n}^{n} c_{k, n} f\left(\frac{k_{1}}{2 n}, \frac{k_{2}}{2 n}\right), \quad \forall f \in \mathcal{H}_{2 n-1}^{*},
$$

where $c_{k, n}=1$ if $k \in(-n, n)^{2}, c_{k, n}=1 / 2$ if either $k_{1}= \pm n$ or $k_{2}= \pm n$ but not both, and $c_{k, n}=1 / 4$ if $k=( \pm n, \pm n)$. The second one is obtained by applying (2.6) to the function $f\left(\cdot+\frac{1}{4 n}\right)$ and using the periodicity of $f$ in the integral,

$$
\int_{\left[-\frac{1}{2}, \frac{1}{2}\right]^{2}} f(x) d x=\frac{1}{4 n^{2}} \sum_{k_{1}=-n}^{n-1} \sum_{k_{2}=-n}^{n-1} f\left(\frac{k_{1}+\frac{1}{2}}{2 n}, \frac{k_{2}+\frac{1}{2}}{2 n}\right), \quad f \in \mathcal{H}_{2 n-1}^{*} .
$$

The fact that the set of nodes in either (2.7) or (2.8) is invariant under the group $\mathbb{Z}_{2}^{2}$ (sign changes) allows us to derive cubature formulas for product cosine and produce sine functions. Let $\mathcal{T} C_{n}:=\operatorname{span}\left\{\cos 2 \pi k_{1} x_{1} \cos 2 \pi k_{2} x_{2}: 0 \leq k_{1}, k_{2} \leq n\right\}$ and $\mathcal{T} S_{n}:=\operatorname{span}\left\{\sin 2 \pi k_{1} x_{1} \sin 2 \pi k_{2} x_{2}: 1 \leq k_{1}, k_{2} \leq n\right\}$, which consist of functions in $\mathcal{H}_{n}^{*}$ that are invariant or anti-invariant under $\mathbb{Z}_{2}^{2}$, respectively.

Stage 3. Restricting (2.8) to $\mathcal{T} C_{2 n-1}$, we obtain a trigonometric cubature,

$$
\int_{\left[0, \frac{1}{2}\right]^{2}} f(x) d x=\frac{1}{4 n^{2}} \sum_{k_{1}=0}^{n-1} \sum_{k_{2}=0}^{n-1} f\left(\frac{k_{1}+\frac{1}{2}}{2 n}, \frac{k_{2}+\frac{1}{2}}{2 n}\right), \quad \forall f \in \mathcal{T} C_{2 n-1},
$$

whereas restricting (2.7) to $\mathcal{T} C_{2 n-1}$ gives another trigonometric cubature for $\mathcal{T} C_{2 n-1}$. Furthermore, restricting (2.7) or (2.8) to $\mathcal{T} S_{2 n-1}$ leads to cubature for $\mathcal{T} S_{2 n-1}$.

The Chebyshev polynomials of the first and the second kind are defined, respectively, by $T_{n}(\xi)=\cos 2 \pi n \theta$ and $U_{n}(\xi)=\sin 2 \pi(n+1) \theta / \sin 2 \pi \theta$, where $\xi=\cos 2 \pi \theta$ with $0 \leq \theta \leq 1 / 2$. These are orthogonal polynomials with respect to $w_{0}(\xi):=$ $\left(1-\xi^{2}\right)^{-1 / 2}$ and $w_{1}(\xi):=\left(1-\xi^{2}\right)^{1 / 2}$, respectively, on $[-1,1]$. Consequently, under the change of variables

$$
\left(x_{1}, x_{2}\right) \mapsto\left(y_{1}, y_{2}\right)=\left(\cos 2 \pi x_{1}, \cos 2 \pi x_{2}\right) \in[-1,1]^{2},
$$

the space $\mathcal{T} C_{n}$ is mapped into the product space $\Pi_{n} \times \Pi_{n}$, where $\Pi_{n}$ denotes the space of algebraic polynomials of one variable, and $\mathcal{T} S_{n}$ is mapped into $\Pi_{n-1} \times \Pi_{n-1}$.

Stage 4. Under the map $x \mapsto y$ of (2.10), the cubature (2.9) becomes

$$
\frac{1}{\pi^{2}} \int_{[-1,1]^{2}} f(y) W_{0}(y) d y=\frac{1}{n^{2}} \sum_{k_{1}=0}^{n-1} \sum_{k_{2}=0}^{n-1} f\left(\cos \frac{\pi\left(2 k_{1}+1\right)}{2 n}, \cos \frac{\pi\left(2 k_{2}+1\right)}{2 n}\right),
$$

for $f \in \Pi_{2 n-1} \times \Pi_{2 n-1}$, where $W_{0}(y)=w_{0}\left(y_{1}\right) w_{0}\left(y_{2}\right)$, which is in fact the product Chebyshev-Gauss cubature of the first kind. Applying the same procedure on the cubature (2.7), we obtain the product Chebyshev-Gauss-Lobatto cubature. Furthermore, if we apply this procedure on the cubature for $\mathcal{T} S_{2 n-1}$ that were mentioned in Stage 3, we obtain the product cubature for the product Chebyshev weight $W_{1}(y)=w_{1}\left(y_{1}\right) w_{1}\left(y_{2}\right)$ of the second kind. 


\section{DisCRETE FouRIER ANALYSIS ON PLANER DOMAINS}

We now apply the general theory in Section 2.1 on the non-classical choices of lattices. The guideline of our choices is the program that we outlined for the classical case in subsection 2.2. We list the cases according to the shapes of the fundamental domains of lattices. For example, the classical case in Section 2.2 is Square-Square. The main ones that we consider are the regular domains such as square, rhombus, and regular hexagon, which are depicted below.
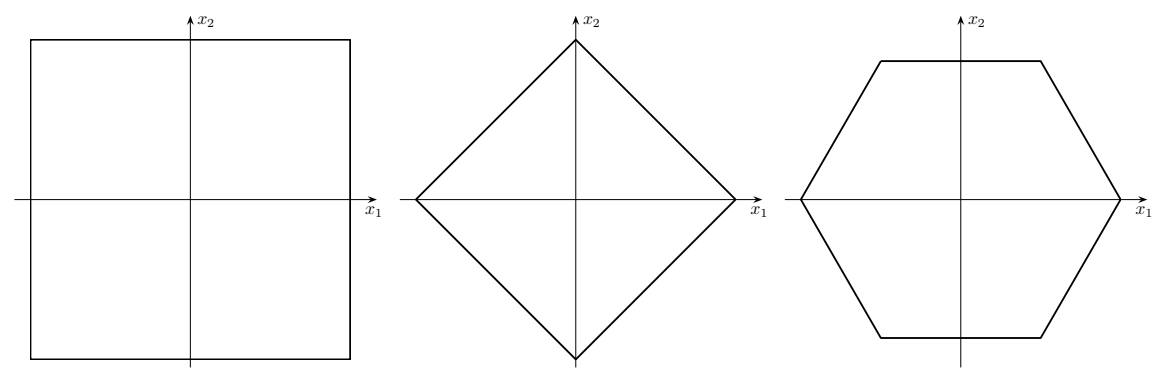

FiguRE 1. Square, rhombus, regular hexagon

3.1. Square-Rhombus. In this case we choose $A=I$ with $\Omega_{A}=\left[-\frac{1}{2}, \frac{1}{2}\right)^{2}$ being the square and choose $B=n R$, where $R \mathbb{Z}^{2}$ has rhombic as its fundamental domain,

$$
R:=\left[\begin{array}{cc}
1 & 1 \\
-1 & 1
\end{array}\right] \quad \text { with } \quad \Omega_{B}=\left\{x \in \mathbb{R}^{2}:-n \leq x_{1}+x_{2}<n,-n \leq x_{2}-x_{1}<n\right\} .
$$

This case was studied in [8]. We shall be brief. Here $\Lambda_{N}=\Lambda_{N}^{\dagger}=: \Lambda_{n}$, where

$$
\Lambda_{n}=\left\{j \in \mathbb{Z}^{2}:-n \leq j_{2} \pm j_{1}<n\right\} \quad \text { and } \quad \Lambda_{n}^{*}:=\left\{j \in \mathbb{Z}^{2}:-n \leq j_{2} \pm j_{1} \leq n\right\} .
$$

The set $\Lambda_{n}$ is not symmetric on $[-n, n]^{2}$ but $\Lambda_{n}^{*}$ is. The cardinality of $\Lambda_{n}$ is $\left|\Lambda_{n}\right|=2 n^{2}$. We follow the program in Section 2.2: In Stage 1 we deduce a cubature from Theorem 2.2, which has nodes indexed by $\Lambda_{n}$, then in Stage 2 we derive a cubature by periodicity that has nodes indexed by $\Lambda_{n}^{*}$. By considering functions that are even in both variables, we deduce in Stage 3 a cubature for trigonometric polynomials, which we state as follows. Changing variables from $j$ to $k=2 n B^{-\operatorname{tr}} j$, or $k_{1}=j_{1}+j_{2}$ and $k_{2}=j_{2}-j_{1}$, it follows easily that $j \in \Lambda_{n}^{*}$ is equivalent to

$$
k \in X_{n}^{*}=\left\{2 k:-\frac{n}{2} \leq k_{1}, k_{2} \leq \frac{n}{2}\right\} \cup\left\{2 k+1:-\frac{n+1}{2} \leq k_{1}, k_{2} \leq \frac{n-1}{2}\right\} .
$$

Let $X_{n}^{\circ}, X_{n}^{e}$ and $X_{n}^{v}$ denote the set of points in $X_{n}^{*}$ that lie in the interior, the edges excluding corners, and the corners of $[-n, n]^{2}$, respectively.

Throughout the rest of the paper, we will adopt the convention that $X^{\circ}, X^{e}$ and $X^{v}$ are subsets of $X$ defined as above, whenever the domain to which the interior, edges and corners relate to is clear.

Theorem 3.1. For $n \geq 2$, the cubature formula

$$
\int_{\left[-\frac{1}{2}, \frac{1}{2}\right]^{2}} f(x) d x=\frac{1}{2 n^{2}} \sum_{k \in X_{n}^{*}} c_{k}^{(n)} f\left(\frac{k}{2 n}\right) \quad \text { with } \quad c_{k}^{(n)}= \begin{cases}1, & k \in X_{n}^{\circ} \\ \frac{1}{2}, & k \in X_{n}^{e} \\ \frac{1}{4}, & k \in X_{n}^{v}\end{cases}
$$

is exact for $f \in \mathcal{T}_{2 n-1}^{*}$, where $\mathcal{T}_{m}^{*}:=\operatorname{span}\left\{\mathrm{e}^{2 \pi i k \cdot x}: k \in \Lambda_{m}^{*}\right\}$. 
The index set $\Lambda_{n}^{*}$ is most suitable for dealing with algebraic polynomials. In fact, under the change of variables $x \mapsto y$ in (2.10), the space $\mathcal{T} C_{m}^{*}$ becomes the space $\Pi_{m}^{2}$ of algebraic polynomial of total degree $m$ and the cubature (3.1) becomes a cubature for the product Chebyshev weight $W_{0}(y)$ that is exact for $\Pi_{2 n-1}^{2}$. Let

$$
\Xi_{n}:=\left\{\left(2 k_{1}, 2 k_{2}\right): 0 \leq k_{1}, k_{2} \leq \frac{n}{2}\right\} \cup\left\{\left(2 k_{1}+1,2 k_{2}+1\right): 0 \leq k_{1}, k_{2} \leq \frac{n-1}{2}\right\} .
$$

Then, in Stage 4, (3.1) becomes the following:

Theorem 3.2. Let $z_{k}=\left(\cos \frac{k_{1} \pi}{n}, \cos \frac{k_{2} \pi}{n}\right)$. Then the cubature below is exact for $f \in \Pi_{2 n-1}^{2}$,

$$
\frac{1}{\pi^{2}} \int_{[-1,1]^{2}} f(y) W_{0}(y) d y=\frac{1}{2 n^{2}} \sum_{k \in \Xi_{n}} \lambda_{k}^{(n)} f\left(z_{k}\right), \quad \lambda_{k}^{(n)}:= \begin{cases}4, & k \in \Xi_{n}^{\circ}, \\ 2, & k \in \Xi_{n}^{e}, \\ 1, & k \in \Xi_{n}^{v} .\end{cases}
$$

The cardinality of $\Xi_{n}$ is $\left|\Xi_{n}\right|=\frac{n(n+1)}{2}+\left\lfloor\frac{n}{2}\right\rfloor+1$, which is just one more than the theoretic lower bound for all such cubature ([3, 12]). The formula (3.2) first appeared in 20, where it is constructed by considering the common zeros of orthogonal polynomials of two variables; see also [13. We can also derive similarly cubature for the product Chebyshev weight $W_{1}(y)$ of the second kind.

The Lagrange interpolation polynomials based on the points in $\Xi_{n}$ were defined and studied in 21. The result there has also been recovered in [8], with (2.3) as the starting point, by utilizing the discrete Fourier analysis.

3.2. Rhombic-Square. In this case we choose $A=R$ with fundamental domain $\Omega_{R}=\left\{x \in \mathbb{R}^{2}:-1 \leq x_{2} \pm x_{1}<1\right\}$, the rhombic, and $B=n I$. Again write $\Lambda_{n} \ldots$ in place of $\Lambda_{N} \ldots$. It is then easy to verify that $\Lambda_{n}=\Lambda_{n}^{\dagger}$ with

$$
\Lambda_{n}=\left\{j \in \mathbb{Z}^{2}:-n \leq k_{2} \pm k_{1}<n\right\} \quad \text { and } \quad \Lambda_{n}^{*}:=\left\{j \in \mathbb{Z}^{2}:-n \leq k_{2} \pm k_{1} \leq n\right\} .
$$

Furthermore, the space of exponential functions $\mathcal{H}_{n}$ is given by

$$
\mathcal{H}_{n}=\left\{\mathrm{e}^{\pi i\left(\left(k_{1}+k_{2}\right) x_{1}+\left(k_{2}-k_{1}\right) x_{2}\right)}: k \in \Lambda_{n}\right\}
$$

and $\mathcal{H}_{n}^{*}$ is likewise defined in terms of $\Lambda_{n}^{*}$. Changing variables shows that

$$
\mathcal{H}_{n}^{*}:=\left\{\mathrm{e}^{\pi i\left(j_{1} x_{1}+j_{2} x_{2}\right)}:-n \leq j_{1}, j_{2} \leq n, j_{1} \equiv j_{2} \quad(\bmod 2)\right\} .
$$

Following the program in Section 2.1, it is easy to see that the cubature in Stage 2 that has symmetric set of nodes, indexed by $\Lambda_{n}^{*}$, takes the form

$$
\frac{1}{2} \int_{\Omega_{R}} f(x) d x=\frac{1}{2 n^{2}} \sum_{k \in \Lambda_{n}^{*}} c_{k, n} f\left(\frac{k}{n}\right), \quad f \in \mathcal{H}_{2 n-1}^{*}, \quad c_{k, n}= \begin{cases}1, & k \in \Lambda_{n}^{\circ}, \\ \frac{1}{2}, & k \in \Lambda_{n}^{e}, \\ \frac{1}{4}, & k \in \Lambda_{n}^{v}\end{cases}
$$

The subspace of functions in $\mathcal{H}_{n}^{*}$ that are even in both variables becomes

$$
\mathcal{T}_{n}:=\operatorname{span}\left\{\cos \pi j_{1} x_{1} \cos \pi j_{2} x_{2}: 0 \leq j_{1}, j_{2} \leq n, j_{1} \equiv j_{2} \quad(\bmod 2)\right\} .
$$

For functions in $\mathcal{T}_{2 n-1}$, we only need to consider the triangle $T_{R}:=\{x: 0 \leq$ $\left.x_{1}, x_{2}, x_{1}+x_{2} \leq 1\right\}$. Thus, in Stage 3, cubature (3.3) becomes

$$
2 \int_{T_{R}} f(x) d x=\frac{1}{2 n^{2}} \sum_{k \in \Xi_{n}} \lambda_{k, n} f\left(\frac{k}{n}\right), \quad f \in \mathcal{T}_{2 n-1},
$$

where $T_{R}$ is the triangular domain $\Xi_{n}=\left\{\left(k_{1}, k_{2}\right): 0 \leq k_{1}, k_{2}, k_{1}+k_{2} \leq n\right\}$, and $\lambda_{k, n}=4$ if $k \in \Xi_{n}^{\circ}, \lambda_{k, n}=2$ if $k \in \Xi_{n}^{e}, \lambda_{(0,0), n}=1$, and $\lambda_{(n, 0), n}=\lambda_{(0, n), n}=1 / 2$. 
Under the mapping $x \mapsto y=\left(\cos \pi x_{1}, \cos \pi x_{2}\right)$, the boundary $x_{1}+x_{2}=1$ of the triangle $T_{R}$ is mapped onto $y_{1}+y_{2}=0$, so that $T_{R}$ is mapped onto the triangle $T_{S}=\left\{\left(y, y_{2}\right):-1 \leq y_{1}, y_{2} \leq 1, y_{1}+y_{2} \geq 0\right\}$, which is half of the square $[-1,1]^{2}$. The cubature (3.5) in Stage 4 becomes a cubature with respect to the product Chebyshev weight $W_{0}$ over $T_{S}$ that is exact for the subspace of polynomials $\Pi_{2 n-1}^{*}=\left\{T_{k_{1}}\left(x_{1}\right) T_{k_{2}}\left(x_{2}\right): 0 \leq k_{1}, k_{2} \leq 2 n-1, k_{1} \equiv k_{2}(\bmod 2)\right\}$, the image of $\mathcal{T}_{2 n-1}$ under the same mapping. Since $\Pi_{n}^{*}$ does not contain polynomials of total degree, we shall not write this cubature explicitly out. It is easy to see, however, that this cubature is in fact half of the product Chebyshev-Gaussian-Lobatto cubature, in the sense that its domain is half and it is exact for half of the polynomials of the latter cubature.

3.3. Rhombic-Rhombic. Here we choose $A=R$ and $B=n R^{-\operatorname{tr}}=\frac{n}{2} A$, so that $N=B^{\text {tr }} A=n I$ have integer entries. Then $\Omega_{A}=\Omega_{R}$ as in the previous case. Again denote $\Lambda_{N}, \ldots$ by $\Lambda_{n}, \ldots$. It is easy to see that $\Lambda_{n}=\Lambda_{n}^{\dagger}$ with

$$
\Lambda_{n}=\left\{j \in \mathbb{Z}^{2}:-\frac{n}{2} \leq-j_{1}, j_{2}<\frac{n}{2}\right\} \quad \text { and } \quad \Lambda_{n}^{*}:=\left\{j \in \mathbb{Z}^{2}:-\frac{n}{2} \leq j_{1}, j_{2} \leq \frac{n}{2}\right\} .
$$

Moreover, the space of exponential functions $\mathcal{H}_{n}$ is given by, as in Section 3.2,

$$
\mathcal{H}_{n}=\left\{\mathrm{e}^{\pi i\left(\left(k_{1}+k_{2}\right) x_{1}+\left(k_{2}-k_{1}\right) x_{2}\right)}: k \in \Lambda_{n}\right\}
$$

and $\mathcal{H}_{n}^{*}$ is likewise defined with $\Lambda_{n}$ replaced by $\Lambda_{n}^{*}$. In this case, the cubature derived from Theorem 2.2, in Stage 1, takes the form

$$
\frac{1}{2} \int_{\Omega_{R}} f(x) d x=\frac{1}{n^{2}} \sum_{k \in \Lambda_{n}} f\left(\frac{k_{1}+k_{2}}{n}, \frac{k_{2}-k_{1}}{n}\right), \quad \forall f \in \mathcal{H}_{2 n-1}^{*} .
$$

The set of nodes of this cubature is on $\Omega_{R}$, and it contains no points on the boundary of $\Omega_{R}$ when $n$ is an odd integer, whereas it contains points on half of the boundary of $\Omega_{R}$ when $n$ is an even integer. In the latter case, we can again derive a cubature, exact for $\mathcal{H}_{2 n-1}^{*}$, that has notes indexed by $\Lambda_{n}^{*}$ as in Stage 2. Let us consider, however, only the case of $n$ being an odd integer below. As can be seen upon changing variables $j_{1}=k_{1}+k_{2}$ and $j_{2}=k_{2}-k_{1}$, the subspace of functions in $\mathcal{H}_{n}^{*}$ that are even in both variables is exactly $\mathcal{T}_{n}$ in (3.4). Thus, just like in the case of Rhombic-Square, restricting (3.6) to functions in $\mathcal{H}_{2 n-1}^{*}$ that are even in both variables, we deduce a cubature of Stage 3 on the triangle $T_{R}$,

$$
2 \int_{T_{R}} f(x) d x=\frac{1}{n^{2}} \sum_{k \in \Xi_{n}} \lambda_{k, n} f\left(\frac{k_{1}+k_{2}}{n}, \frac{k_{2}-k_{1}}{n}\right), \quad \forall f \in \mathcal{T}_{2 n-1},
$$

where $\Xi_{n}=\left\{\left(k_{1}, k_{2}\right): 0 \leq k_{1}, k_{2}, k_{1}+k_{2} \leq \frac{n}{2}\right\} ; \lambda_{k, n}=4$ if $k \in \Xi_{n}^{\circ}, \lambda_{k, n}=2$ if either $k_{1}=0$ or $k_{2}=0$ or $k_{1}+k_{2}=\frac{n}{2}$ but not both (i.e., $k \in \Xi_{n}^{e}$ ), and $\lambda_{(0,0), n}=1$, $\lambda_{(0, n), n}=1$.

Finally, under the mapping $x \mapsto y=\left(\cos \pi x_{1}, \cos \pi x_{2}\right)$, the cubature (3.7) becomes a cubature with respect to the product Chebyshev weight $W_{0}$ over the triangle domain $T_{S}$ for the polynomial subspace $\Pi_{2 n-1}^{*}$ defined in the previous subsection. This cubature is exactly half of the algebraic cubature in the Square-Rhombic case. 
3.4. Hexagon-Hexagon. In this case we choose $A=H$ and $B=\frac{n}{2} H$, where

$$
H=\left(\begin{array}{cc}
\sqrt{3} & 0 \\
-1 & 2
\end{array}\right) \quad \text { with } \quad \Omega_{H}=\left\{x \in \mathbb{R}^{2}:-1 \leq x_{2}, \frac{\sqrt{3} x_{1}}{2} \pm \frac{x_{2}}{2}<1\right\} .
$$

This case was studied in [7. We shall be brief, but recall necessary definitions that are needed in the following subsection. As shown in 7, 19, it is more convenient to use homogeneous coordinates $\left(t_{1}, t_{2}, t_{3}\right)$ defined by

$$
\left(\begin{array}{l}
t_{1} \\
t_{2} \\
t_{3}
\end{array}\right)=\left(\begin{array}{cc}
\frac{\sqrt{3}}{2} & -\frac{1}{2} \\
0 & 1 \\
-\frac{\sqrt{3}}{2} & -\frac{1}{2}
\end{array}\right)\left(\begin{array}{l}
x_{1} \\
x_{2}
\end{array}\right):=E x,
$$

which satisfies $t_{1}+t_{2}+t_{3}=0$. We adopt the convention of using bold letters, such as $\mathbf{t}$ to denote points in homogeneous coordinates. We define by

$$
\mathbb{R}_{H}^{3}:=\left\{\mathbf{t}=\left(t_{1}, t_{2}, t_{3}\right) \in \mathbb{R}^{3}: t_{1}+t_{2}+t_{3}=0\right\} \quad \text { and } \quad \mathbb{H}:=\mathbb{Z}^{3} \cap \mathbb{R}_{H}^{3}
$$

the spaces of points and integers in homogeneous coordinates, respectively. In such coordinates, the hexagon $\Omega_{H}$ becomes

$$
\Omega=\left\{\mathbf{t} \in \mathbb{R}_{H}^{3}:-1 \leq t_{1}, t_{2},-t_{3}<1\right\},
$$

which is the intersection of the plane $t_{1}+t_{2}+t_{3}=0$ with the cube $[-1,1]^{3}$. The index sets $\Lambda_{n}$ and $\Lambda_{n}^{\dagger}$ satisfy $\Lambda_{n}=\Lambda_{n}^{\dagger}=\mathbb{H}_{n}$, where

$\mathbb{H}_{n}:=\left\{\mathbf{j} \in \mathbb{H}:-n \leq j_{1}, j_{2},-j_{3}<n\right\} \quad$ and $\quad \mathbb{H}_{n}^{*}:=\left\{\mathbf{j} \in \mathbb{H}^{*}:-n \leq j_{1}, j_{2},-j_{3} \leq n\right\}$

Furthermore, since, for $k=\left(k_{1}, k_{2}\right), k^{\text {tr }} H^{-1} x=\frac{1}{3} \mathbf{k} \cdot \mathbf{t}$ with $\mathbf{k}=\left(k_{1}, k_{2},-k_{1}-k_{2}\right)^{\operatorname{tr}} \in$ $\mathbb{H}$, the exponential functions and the space $\mathcal{H}_{N}$ become

$$
\phi_{\mathbf{k}}(\mathbf{t}):=\mathrm{e}^{\frac{2 \pi i}{3} \mathbf{k}^{\mathrm{tr}} \mathbf{t}} \quad \text { and } \quad \mathcal{H}_{n}:=\left\{\phi_{\mathbf{k}}: \mathbf{k} \in \mathbb{H}_{n}\right\} .
$$

In homogeneous coordinates, $x \equiv y(\bmod H)$ becomes $\mathbf{t} \equiv \mathbf{s}(\bmod 3)$, which is defined by $t_{1}-s_{1} \equiv t_{2}-s_{2} \equiv t_{3}-s_{3}(\bmod 3)$, so that $f$ periodic in $H$, i.e. $f(x+H)=f(x)$, becomes $f(\mathbf{t})=f(\mathbf{t}+\mathbf{j})$ whenever $\mathbf{j} \equiv 0(\bmod 3)$.

In this case, the cubature derived from Theorem 2.2 in Stage 1 has nodes over $\left\{\frac{\mathbf{j}}{n}: \mathbf{k} \in \mathbb{H}_{n}\right\}$, from which we derive another cubature, the set of nodes of which is symmetric and indexed by $\mathbb{H}_{n}^{*}$, as in Stage 2:

Theorem 3.3. The following cubature is exact for $f \in \mathcal{H}_{2 n-1}^{*}$,

$$
\frac{1}{|\Omega|} \int_{\Omega} f(\mathbf{t}) d \mathbf{t}=\frac{1}{3 n^{2}} \sum_{\mathbf{j} \in \mathbb{H}_{n}^{*}} c_{\mathbf{j}}^{(n)} f\left(\frac{\mathbf{j}}{n}\right), \quad c_{\mathbf{j}}^{(n)}= \begin{cases}1, & \mathbf{j} \in \mathbb{H}_{n}^{\circ}, \\ \frac{1}{2}, & \mathbf{j} \in \mathbb{H}_{n}^{e}, \\ \frac{1}{3}, & \mathbf{j} \in \mathbb{H}_{n}^{v},\end{cases}
$$

The group of isometries of the hexagon lattice is generated by the reflections in the edges of the equilateral triangles inside the regular hexagon, which is the reflection group $\mathcal{A}_{2}$. By considering the invariant and anti-invariant functions under $\mathcal{A}_{2}$ in the space $\mathcal{H}_{n}$, we end up with functions that are analogues of cosine and sine functions on an equilateral triangle, and the cubature (3.9) becomes a cubature on the triangle for such functions. To be more precise, we choose the triangle as

$$
\Delta:=\left\{\left(t_{1}, t_{2}, t_{3}\right): t_{1}+t_{2}+t_{3}=0,0 \leq t_{1}, t_{2},-t_{3} \leq 1\right\} .
$$

The region $\Delta$ and its relative position in the hexagon are depicted in Figure 2 , where the points are labeled in homogeneous coordinates. The generalized cosine, 

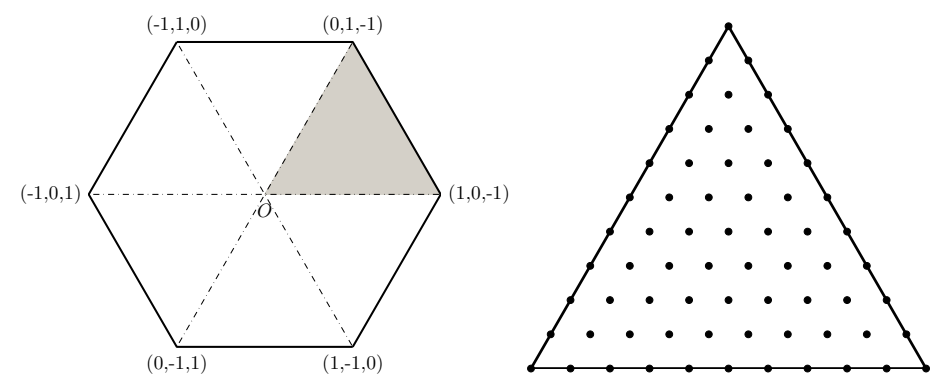

Figure 2. The fundamental triangle of the regular hexagon.

$\mathrm{TC}_{\mathbf{k}}$, and the generalized sine, $\mathrm{TS}_{\mathbf{k}}$, are defined in terms of

$$
\mathcal{P}^{+} f(\mathbf{t}):=\sum_{\sigma \in \mathcal{A}_{2}} f(\mathbf{t} \sigma) \quad \text { and } \quad \mathcal{P}^{-} f(\mathbf{t}):=\sum_{\sigma \in \mathcal{A}_{2}} \operatorname{sign}(\sigma) f(\mathbf{t} \sigma)
$$

as $\mathrm{TC}_{\mathbf{k}}(\mathbf{t}):=\mathcal{P}^{+} \phi_{\mathbf{k}}(\mathbf{t})$ and $\mathrm{TS}_{\mathbf{k}}(\mathbf{t}):=\mathcal{P}^{-} \phi_{\mathbf{k}}(\mathbf{t})$, respectively; more explicitly,

$$
\begin{aligned}
\mathrm{TC}_{\mathbf{k}}(\mathbf{t})= & \frac{1}{3}\left[\mathrm{e}^{\frac{i \pi}{3}\left(k_{2}-k_{3}\right)\left(t_{2}-t_{3}\right)} \cos k_{1} \pi t_{1}+\mathrm{e}^{\frac{i \pi}{3}\left(k_{2}-k_{3}\right)\left(t_{3}-t_{1}\right)} \cos k_{1} \pi t_{2}\right. \\
& \left.+\mathrm{e}^{\frac{i \pi}{3}\left(k_{2}-k_{3}\right)\left(t_{1}-t_{2}\right)} \cos k_{1} \pi t_{3}\right], \quad \mathbf{k} \in \Lambda, \\
\mathrm{TS}_{\mathbf{k}}(\mathbf{t})= & \frac{1}{3}\left[\mathrm{e}^{\frac{i \pi}{3}\left(k_{2}-k_{3}\right)\left(t_{2}-t_{3}\right)} \sin k_{1} \pi t_{1}+\mathrm{e}^{\frac{i \pi}{3}\left(k_{2}-k_{3}\right)\left(t_{3}-t_{1}\right)} \sin k_{1} \pi t_{2}\right. \\
& \left.+\mathrm{e}^{\frac{i \pi}{3}\left(k_{2}-k_{3}\right)\left(t_{1}-t_{2}\right)} \sin k_{1} \pi t_{3}\right], \quad \mathbf{k} \in \Lambda^{\circ},
\end{aligned}
$$

where $\Lambda:=\left\{\mathbf{k} \in \mathbb{H}: k_{1} \geq 0, k_{2} \geq 0, k_{3} \leq 0\right\}$ and $\Lambda^{\circ}$ is the interior of $\Lambda$. These functions are orthogonal with respect to the integral over $\Delta$, and they are elements of $\mathcal{H}_{n}$ that invariant and anti-invariant under $\mathcal{A}_{2}$, respectively. The cubature (3.9) when restrict to invariant functions becomes, as in Stage 3, the following:

Theorem 3.4. Let $\mathcal{T} C_{n}:=\operatorname{span}\left\{\mathrm{TC}_{\mathbf{k}}: \mathbf{k} \in \Lambda_{n}\right\}$. The cubature below is exact for all $f \in \mathcal{T} C_{2 n-1}$,

$$
2 \int_{\Delta} f\left(t_{1}, t_{2}\right) d t_{1} d t_{2}=\frac{1}{3 n^{2}} \sum_{j_{1}=0}^{n} \sum_{j_{2}=0}^{j_{1}} \lambda_{\mathbf{j}}^{(n)} f\left(\frac{j_{1}}{n}, \frac{j_{2}}{n}\right), \quad \lambda_{\mathbf{j}}^{(n)}:= \begin{cases}6, & \mathbf{j} \in \Lambda_{n}^{\circ} \\ 3, & \mathbf{j} \in \Lambda_{n}^{\mathrm{e}} \\ 1, & \mathbf{j} \in \Lambda_{n}^{\mathrm{v}}\end{cases}
$$

The nodes of the cubature (3.14) are equally spaced points in $\Delta$ (Figure 2).

The generalized cosine and sine functions can be mapped into algebraic polynomials of two variables under the following mapping,

$$
\begin{aligned}
& x=\frac{4}{3} \cos \frac{\pi}{3}\left(t_{2}-t_{1}\right) \cos \frac{\pi}{3}\left(t_{3}-t_{2}\right) \cos \frac{\pi}{3}\left(t_{1}-t_{3}\right)-\frac{1}{3}, \\
& y=\frac{4}{3} \sin \frac{\pi}{3}\left(t_{2}-t_{1}\right) \sin \frac{\pi}{3}\left(t_{3}-t_{2}\right) \sin \frac{\pi}{3}\left(t_{1}-t_{3}\right),
\end{aligned}
$$

which are the real and imaginary part of $\mathrm{TC}_{0,1,-1}(\mathbf{t})$, the first non trivial generalized cosine function. Under this mapping, we call the polynomials

$$
T_{k}^{m}(x, y):=\mathrm{TC}_{k, m-k,-m}(\mathbf{t}) \quad \text { and } \quad U_{k}^{m}(x, y):=\frac{\mathrm{TS}_{k+1, m-k+1,-m-2}(\mathbf{t})}{\mathrm{TS}_{1,1,-2}(\mathbf{t})},
$$


where $0 \leq k \leq m$, generalized Chebyshev polynomials of the first and the second kind, respectively. They are algebraic polynomials of total degree $n$ and are orthogonal polynomials with respect to the weight function $w_{-\frac{1}{2}}(x, y)$ and $w_{\frac{1}{2}}(x, y)$, respectively, where $w_{\alpha}(x, y)$ is defined by

$$
w_{\alpha}(x, y)=\frac{4^{\alpha}}{27^{\alpha}} \pi^{4 \alpha}\left[-3\left(x^{2}+y^{2}+1\right)^{2}+8\left(x^{3}-3 x y^{2}\right)+4\right]^{\alpha},
$$

and the integral domain is the region $\Delta^{*}$ bounded by the Steiner's hypocycloid, depicted in Figure 3, which is the region on which $w_{\alpha}(x, y)$ is positive. These

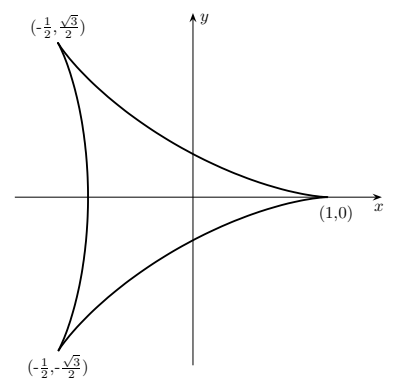

Figure 3 . The region $\Delta^{*}$ bounded by Steiner's hypocycloid.

polynomials were first studied in [6. As in Stage 4, the cubature (3.14) under the change of variable (3.15) becomes a cubature for $w_{-\frac{1}{2}}(x, y) d x d y$ on $\Delta^{*}$ that has $\operatorname{dim} \Pi_{n}^{2}$ nodes and is exact for algebraic polynomials of degree $2 n-1$. Furthermore, we can derive a cubature from (3.9) for anti-invariant functions in Stage 3, which becomes under (3.15) a cubature for $w_{\frac{1}{2}}(x, y) d x d y$ that has $\operatorname{dim} \Pi_{n-1}^{2}$ nodes and is exact for algebraic polynomials of degree $2 n-1$. The latter one provides an example of a family of Gaussian cubature formulas, a rarity of only the second example known so far (the first one appeared in [17]); see [7] for details. We refer to 3, 16, 18, for the topic of Gaussian cubature.

We now address one question that was not addressed in 7 . Taking the cue form the cubature 2.8 in the Square-Square case, we can apply the cubature derived in Stage 1 on the functions $f(\mathbf{t}+\mathbf{a})$ and then use the hexagonal periodicity of the integral to derive the following cubature in Stage 2,

$$
\frac{1}{|\Omega|} \int_{\Omega} f(\mathbf{t}) d \mathbf{t}=\frac{1}{3 n^{2}} \sum_{\mathbf{j} \in \mathbb{H}_{n}} f\left(\frac{\mathbf{j}}{n}+\mathbf{a}\right), \quad f \in \mathcal{H}_{2 n-1}^{*},
$$

and hope to choose a so that the set of nodes in (3.16) is symmetric. The question is if it is possible to find a a so that the set of nodes has full symmetry of $\mathcal{A}_{2}$.

It is easy to see that if $\mathbf{a}=\left(a_{1}, a_{2},-a_{1}-a_{2}\right) \in \mathbb{R}_{H}^{3}$ satisfies $\left|a_{1}\right|,\left|a_{2}\right| \leq 1 / n$, then the set of nodes of (3.16) will be inside the hexagon $\Omega$, although not symmetric for most of the choices. The two cases that offer the most symmetry are

$$
\mathbf{a}_{1}:=\left(\frac{1}{3 n}, \frac{1}{3 n},-\frac{2}{3 n}\right) \quad \text { and } \quad \mathbf{a}_{2}:=\left(-\frac{1}{3 n},-\frac{1}{3 n}, \frac{2}{3 n}\right),
$$

where, when $\mathbf{a}_{2}$ is used, we need to use the periodicity of $f$ (or congruent relation with respect to $H$ ) to make sure that all points in (3.16) are in $\Omega$. Neither of these two choices, however, offer complete symmetry under the group $\mathcal{A}_{2}$. In Figure 4 , we depict the set of points resulted from these two choices. Each set of the points is 

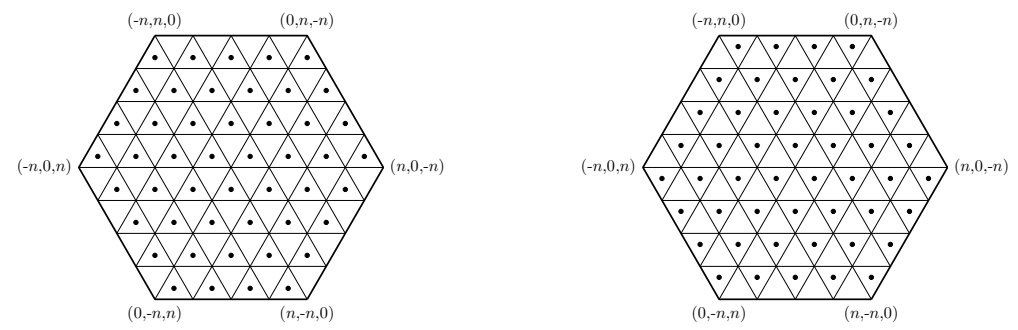

Figure 4. Nodes of (3.16) with $\mathbf{a}_{1}$ (left) and $\mathbf{a}_{2}$ (right)

invariant under a subgroup of $\mathcal{A}_{2}$ of three rotations, but neither is invariant under the group $\mathcal{A}_{2}$. As a result, we cannot restrict the cubature (3.16) with either $\mathbf{a}_{1}$ or $\mathbf{a}_{2}$ to the generalized cosine or sine functions in hopes of obtaining new cubature on the triangle in Stage 3, in contrast to Square-Square case.

The interpolation on the hexagon and on the triangle were studied in [7. In particular, we have Lagrange interpolation based on equally space points on the triangle $\Delta$, which enjoys a closed formula in trigonometric functions and has Lebesgue constant in the order of $(\log n)^{2}$. One can also consider approximation on the hexagon and the triangle $([22])$ for functions that are periodic in $H$.

3.5. Hexagon-Hexagon Transpose. Here we choose $A=H$, the matrix for the hexagon lattice, and choose $B=n H^{-\operatorname{tr}}$ with $n \in \mathbb{Z}$, so that $N=B^{\operatorname{tr}} A=n I$ has all integer entries. The fundamental domain of the lattice $L_{B}$ is given by

$$
\Omega_{B}=\left\{x \in \mathbb{R}^{2}:-\frac{n}{2 \sqrt{3}} \leq x_{1}, \frac{\sqrt{3}}{2} x_{2} \pm \frac{1}{2} x_{1}<\frac{n}{2 \sqrt{3}}\right\} .
$$

3.5.1. Cubature. It is again convenient to use homogeneous coordinates as defined in the previous subsection. The $\Omega_{B}$ is the regular hexagon in Fig. 1 rotated by $90^{\circ}$, as depicted in Figure 2, in which the right hand figure is labeled in homogeneous coordinates. Here the index set $\Lambda_{N}^{\dagger}=\left\{k \in \mathbb{Z}^{2}:-n \leq 2 k_{2}+k_{1}, k_{2}-k_{1}, 2 k_{1}+k_{2}<n\right\}$,
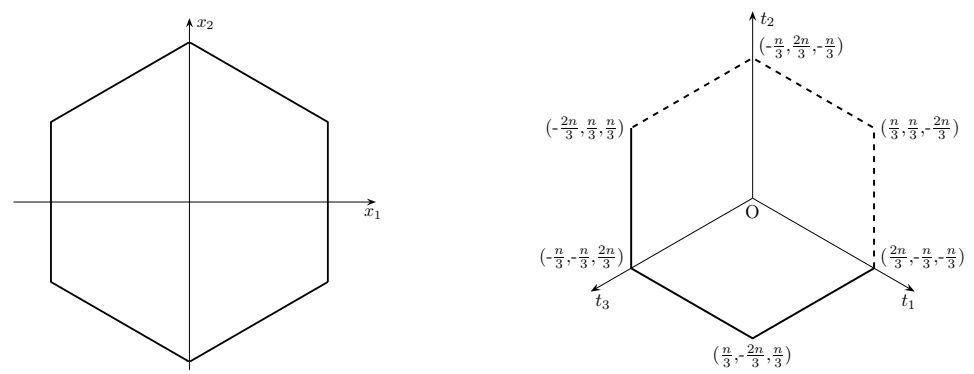

FiguRE 5. The hexagon domain and $\Omega_{B}$

which becomes in homogeneous coordinates $\mathbb{K}_{n}^{\dagger}$ defined by

$$
\mathbb{K}_{n}^{\dagger}:=\left\{\mathbf{j} \in \mathbb{H}:-n \leq j_{2}-j_{1}, j_{1}-j_{3}, j_{2}-j_{3}<n\right\} .
$$

We also have $\Lambda_{N}=\left\{k \in \mathbb{Z}^{2}:-n \leq 2 k_{2}-k_{1}, k_{1}+k_{2}, 2 k_{1}-k_{2}<n\right\}$. Recall that $\mathbf{t} \equiv 0(\bmod 3)$ means, by definition, $t_{1} \equiv t_{2} \equiv t_{3}(\bmod 3)$. It is not hard to see 
that the set $\Lambda_{N}$ becomes, in homogeneous coordinates, $\mathbb{K}_{n}$ defined by

$$
\mathbb{K}_{n}:=\left\{\mathbf{j} \in \mathbb{H}:-n \leq j_{1}, j_{2},-j_{3}<n, \mathbf{j} \equiv 0 \quad(\bmod 3)\right\} .
$$

We also denote by $\mathbb{K}_{n}^{\dagger *}$ and $\mathbb{K}_{n}^{*}$ the sets defined with $\leq$ in place of $<$ in $\mathbb{K}_{n}^{\dagger}$ and $\mathbb{K}_{n}$, respectively. The set $\mathbb{K}_{n}^{*}$ can be obtained form a rotation of $\mathbb{K}_{n}^{\dagger *}$, as shown in the following proposition, which can be easily verified.

Proposition 3.5. For $\mathbf{k}=\left(k_{1}, k_{2}, k_{3}\right) \in \mathbb{H}$, define $\widehat{\mathbf{k}}:=\left(k_{3}-k_{2}, k_{1}-k_{3}, k_{2}-k_{1}\right)$. Then $\frac{\widehat{\mathbf{k}}}{3} \in \mathbb{K}_{n}^{\dagger *}$ if $\mathbf{k} \in \mathbb{K}_{n}^{*}$ and $\widehat{\mathbf{k}} \in \mathbb{K}_{n}^{*}$ if $\mathbf{k} \in \mathbb{K}_{n}^{\dagger *}$.

The finite dimensional space $\mathcal{H}_{n}$ of exponential functions becomes

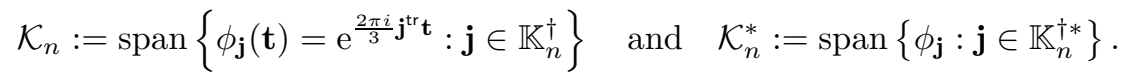

By induction, it follows that $\operatorname{dim} \mathcal{K}_{n}=\left|\mathbb{K}_{n}\right|=n^{2}$ and $\operatorname{dim} \mathcal{K}_{n}^{*}=\left|\mathbb{K}_{n}^{*}\right|=n^{2}+n+1$ if $n=0,2(\bmod 3)$ and $\left|\mathbb{K}_{n}^{*}\right|=n^{2}+n-1$ if $n=1(\bmod 3)$. The two sets $\mathbb{K}_{3 n}^{*}$ and
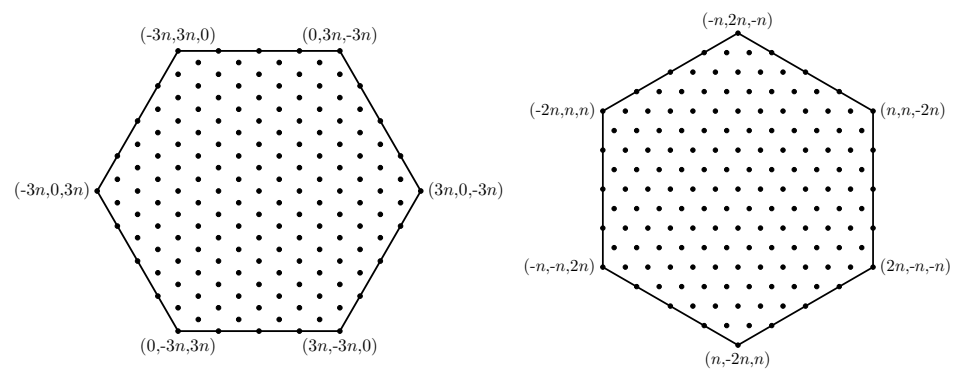

Figure 6 . The set $\mathbb{K}_{3 n}^{*}$ (left) and the set $\mathbb{K}_{3 n}^{\dagger *}$ (right).

$\mathbb{K}_{3 n}^{\dagger *}$ take different shapes, which we depict in Figure [6 Define

$$
\langle f, g\rangle_{n}:=\frac{1}{n^{2}} \sum_{\mathbf{j} \in \mathbb{K}_{n}} f\left(\frac{\mathbf{j}}{n}\right) \overline{f\left(\frac{\mathbf{j}}{n}\right)} \quad \text { and } \quad\langle f, g\rangle_{n}^{*}=\frac{1}{n^{2}} \sum_{\mathbf{j} \in \mathbb{K}_{n}^{*}} c_{\mathbf{j}}^{(n)} f\left(\frac{\mathbf{j}}{n}\right) \overline{f\left(\frac{\mathbf{j}}{n}\right)},
$$

where $c_{\mathbf{j}}^{(n)}$ are as defined in (3.9) with $\mathbb{H}_{n}$ replaced by $\mathbb{K}_{n}$.

Theorem 3.6. For $n \geq 0, \frac{1}{|\Omega|} \int_{\Omega} f(x) \overline{g(x)} d x=\langle f, g\rangle_{n}=\langle f, g\rangle_{n}^{*}$ for $f, g \in \mathcal{K}_{n}$. In particular, $\left\langle\phi_{\mathbf{j}}, \phi_{\mathbf{k}}\right\rangle_{n}^{*}=1$ if $\hat{\mathbf{j}} \equiv \hat{\mathbf{k}}(\bmod 3 n)$ and $\left\langle\phi_{\mathbf{j}}, \phi_{\mathbf{k}}\right\rangle_{n}^{*}=0$ otherwise, for $\mathbf{k}, \mathbf{j} \in \mathbb{K}_{n}$. Moreover, we have the cubature

$$
\frac{1}{|\Omega|} \int_{\Omega} f(\mathbf{t}) d \mathbf{t}=\frac{1}{n^{2}} \sum_{\mathbf{j} \in \mathcal{K}_{n}^{*}} c_{\mathbf{j}}^{(n)} f\left(\frac{\mathbf{j}}{n}\right), \quad \forall f \in \mathcal{K}_{2 n-1}^{*} .
$$

The part of the theorem on $\langle f, g\rangle_{n}$ is exactly Theorem 2.2, while the part on $\langle f, g\rangle_{n}^{*}$ and the cubature can be proved by periodicity, just like the proof of Theorem 3.3 in [7, upon using the Proposition 3.5. The cubature (3.18) is already one in Stage 2; we can also derive a cubature with nodes indexed by $\mathbb{K}_{n}$ as in Stage 1.

Next we consider the invariant and anti-invariant functions under $\mathcal{A}_{2}$, which are the generalized cosines $T C_{k}$ and the generalized sines $T_{k}$ considered in the previous subsection. By restricting to such functions, we again obtain cubature on the triangle $\Delta$. The index set of the nodes of the cubature, denoted by $\Upsilon$, is

$$
\Upsilon_{n}:=\left\{\mathbf{j} \in \mathbb{H}: 0 \leq j_{1}, j_{2},-j_{3} \leq n, \mathbf{j} \equiv 0 \quad(\bmod 3)\right\}
$$


derived by symmetry from $\mathbb{K}_{n}^{*}$, whereas the index set of the invariant functions being integrated exactly by the cubature, denoted by $\Upsilon^{\dagger}$, is derived from $\mathbb{K}_{n}^{\dagger *}$,

$$
\Upsilon_{n}^{\dagger}=\left\{\mathbf{j} \in \mathbb{H}: 0 \leq j_{1}, j_{2},-j_{3} \leq n, j_{2}-j_{3} \leq n, j_{1}-j_{3} \leq n\right\},
$$

which is inside a quadrilateral; Figure 7 shows its relative position in $\Omega_{B}$. We define
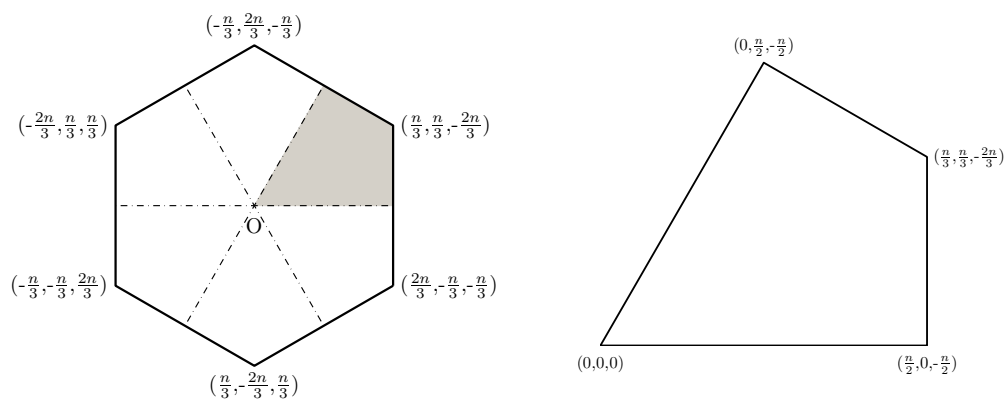

Figure 7 . The fundamental quadrilateral of $\Omega_{B}$ under $\mathcal{A}_{2}$

the following subspaces of trigonometric functions,

$$
\mathcal{T} C_{n}=\operatorname{span}\left\{\mathrm{TC}_{\mathbf{k}}: \mathbf{k} \in \Upsilon_{n}^{\dagger}\right\} \quad \text { and } \quad \mathcal{T} S_{n}=\operatorname{span}\left\{\mathrm{TS}_{\mathbf{k}}: \mathbf{k} \in \Upsilon_{n}^{\dagger \circ}\right\}
$$

The set $\Upsilon_{n}$ takes a symmetric form when $n$ is a multiple of 3 . In Figure (8) we depict the index sets $\Upsilon_{3 n}$ and $\Upsilon_{3 n}^{\dagger}$.
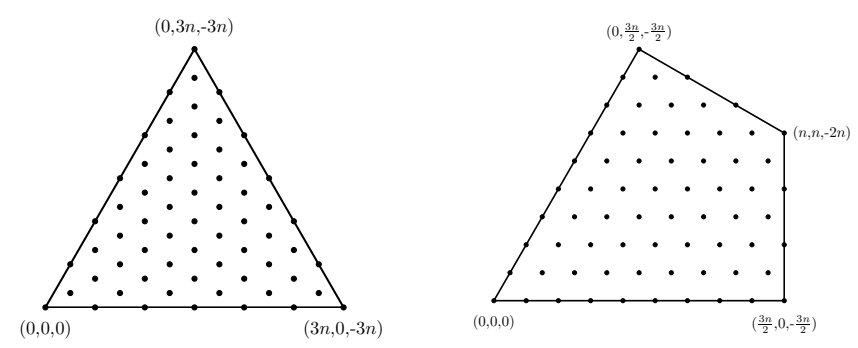

FiguRE 8. the index sets $\Upsilon_{3 n}$ (left) and $\Upsilon_{3 n}^{\dagger}$ (right).

Theorem 3.7. The following cubature is exact for all $f \in \mathcal{T} C_{2 n-1}$,

$$
\frac{1}{|\Delta|} \int_{\Delta} f(\mathbf{t}) d \mathbf{t}=\frac{1}{n^{2}} \sum_{\mathbf{j} \in \Upsilon_{n}} \lambda_{\mathbf{j}}^{(n)} f\left(\frac{\mathbf{j}}{n}\right), \quad \lambda_{\mathbf{j}}^{(n)}:= \begin{cases}6, & \mathbf{j} \in \Upsilon_{n}^{\circ}, \\ 3, & \mathbf{j} \in \Upsilon_{n}^{\mathrm{e}}, \\ 1, & \mathbf{j} \in \Upsilon_{n}^{\mathrm{v}}\end{cases}
$$

The formula (3.19) is derived from (3.18) by using the invariance of the functions in $\mathcal{T} C_{2 n-1}$ and the fact $\Omega=\left(\cup_{\sigma \in \mathcal{A}_{2}}\left\{\mathbf{t} \sigma: \mathbf{t} \in \Delta^{\circ}\right\}\right) \cup\left(\cup_{\sigma \in \mathcal{A}_{2}}\{\mathbf{t} \sigma: \mathbf{t} \in \partial \Delta\}\right)$. As the proof is similar to that of (3.14) in [7, we shall omit the details.

Similarly, we can also derive a cubature for $\mathcal{T} S_{2 n-1}$ based on points in $\Upsilon_{n}^{\circ}$. These are cubature in Stage 3. We note that the set of nodes in (3.19) is different from that in (3.14), see Figure 2 and Figure 8, even though both are on the triangle.

As in the case of Hexagon-Hexagon, we can continue to Stage 4, where the cubature (3.19) is mapped by the change of variables (3.15) to an algebraic cubature 
for $w_{-1 / 2}(x, y) d x d y$ on $\Delta^{*}$, the region bounded by Steiners hypocycloid, which is a cubature exact for all polynomials in $\Pi_{2 n-1}^{2}$ but with many more nodes than the one derived in the Hexagon-Hexagon case.

The set of cubature points in (3.19) and its image in the region bounded by Steiner's hypocycloid have also been studied in [15].

3.5.2. Interpolation. Applying Theorem 2.3 to the current set up, we obtain an interpolation operator $\mathcal{I}_{n} f$ that interpolates $f$ on $\mathbb{K}_{n}$ in the hexagon. We would like to consider interpolation on the triangle based on points in $\Upsilon_{n}$. For this purpose, we first construct a near interpolation operator on the symmetric set of points $\mathbb{K}_{n}^{*}$.

Theorem 3.8. Let $\mathcal{S}_{\mathbf{j}}:=\left\{\mathbf{k} \in \mathbb{K}_{n}^{*}: \mathbf{k} \equiv \mathbf{j}(\bmod 3 n)\right\}$. For $f \in C(\Omega)$, define

$$
\mathcal{I}_{n}^{*} f(\mathbf{t}):=\sum_{\mathbf{j} \in \mathbb{K}_{n}^{*}} f\left(\frac{\mathbf{j}}{n}\right) \Phi_{n}\left(\mathbf{t}-\frac{\mathbf{j}}{n}\right), \quad \Phi_{n}(\mathbf{t}):=\frac{1}{n^{2}} \sum_{\mathbf{j} \in \mathbb{K}_{n}^{\dagger *}} c_{\widehat{\mathbf{j}}}^{(n)} \phi_{\mathbf{j}}(\mathbf{t}) .
$$

Then $\mathcal{I}_{n}^{*} f \in \mathcal{K}_{n}^{*}$ and $\mathcal{I}_{n}^{*} f\left(\frac{\mathbf{j}}{n}\right)=f\left(\frac{\mathbf{j}}{n}\right)$ if $\mathbf{j} \in \mathbb{K}_{n}^{\circ}$, and $\mathcal{I}_{n}^{*} f\left(\frac{\mathbf{j}}{n}\right)=\sum_{\mathbf{k} \in \mathcal{S}_{\mathbf{j}}} f\left(\frac{\mathbf{k}}{n}\right)$ if $\mathbf{j} \in \partial \mathbb{K}_{n}^{*}$, the boundary of $\mathbb{K}_{n}^{*}$. Furthermore, $\Phi_{n}^{*}(\mathbf{t})$ is a real function and it is given by the following formula when $n=0(\bmod 3)$,

$$
\begin{aligned}
\Phi_{n}(\mathbf{t})=\frac{1}{n^{2}}[ & -\frac{1}{2} \sum_{i=1}^{3} \frac{\left(2 \cos \pi s_{i}+\cos \pi t_{i}\right) \sin \pi t_{i} \cos \frac{2 \pi n t_{i}}{3}}{\sin \pi t_{1} \sin \pi t_{2} \sin \pi t_{3}} \\
& \left.-\frac{1}{3}\left(\cos \frac{2 n \pi t_{1}}{3}+\cos \frac{2 n \pi t_{2}}{3}+\cos \frac{2 n \pi s_{3}}{3}\right)\right],
\end{aligned}
$$

where $s_{1}=\frac{t_{3}-t_{2}}{3}, s_{2}=\frac{t_{1}-t_{3}}{3}, s_{3}=\frac{t_{2}-t_{1}}{3}$.

Proof. By Proposition 3.5, $\mathbf{k} \in \mathbb{K}_{n}^{*}$ implies $\frac{\widehat{\mathbf{k}}}{3} \in \mathbb{K}_{n}^{\dagger *}$, and $\mathbf{j} \in \mathbb{K}_{n}^{\dagger *}$ implies $\widehat{\mathbf{j}} \in \mathbb{K}_{n}^{*}$. By homogeneity, $\mathbf{k} \cdot \mathbf{t}=\left(k_{3}-k_{2}\right) s_{1}+\left(k_{1}-k_{3}\right) s_{2}+\left(k_{2}-k_{1}\right) s_{3}=\widehat{\mathbf{k}} \cdot \mathbf{s}$. As a result,

$$
\Phi_{n}(\mathbf{t})=\frac{1}{n^{2}} \sum_{\mathbf{j} \in \mathbb{K}_{n}^{\dagger *}} c_{\hat{\mathbf{j}}}^{(n)} \phi_{\hat{\mathbf{j}}}(\mathbf{s})=\frac{1}{n^{2}} \sum_{\mathbf{j} \in \mathbb{K}_{n}^{*}} c_{\mathbf{j}}^{(n)} \phi_{\mathbf{j}}(\mathbf{s}) .
$$

Consequently, by the definition in (3.17),

$$
\Phi_{n}\left(\frac{\mathbf{k}-\mathbf{j}}{n}\right)=\frac{1}{n^{2}} \sum_{\mathbf{l} \in \mathbb{K}_{n}^{*}} c_{\mathbf{l}}^{(n)} \phi_{\mathbf{l}}\left(\frac{\widehat{\mathbf{k}}-\widehat{\mathbf{j}}}{n}\right)=\frac{1}{n^{2}} \sum_{\mathbf{l} \in \mathbb{K}_{n}^{*}} c_{\mathbf{l}}^{(n)} \phi_{\widehat{\mathbf{k}}-\widehat{\mathbf{j}}}\left(\frac{\mathbf{l}}{n}\right)=\left\langle\phi_{\widehat{\mathbf{k}}}, \phi_{\widehat{\mathbf{j}}}\right\rangle_{n}^{*} .
$$

Thus, by Theorem 3.6, it follows that

$$
\Phi_{n}\left(\frac{\mathbf{k}-\mathbf{j}}{n}\right)=\left\langle\phi_{\widehat{\mathbf{k}}}, \phi_{\widehat{\mathbf{j}}}\right\rangle_{n}^{*}= \begin{cases}1, & \widehat{\mathbf{k}}=\widehat{\mathbf{j}}+n \mathbf{l}, \mathbf{l} \in \mathbb{H}, \\ 0, & \text { otherwise }\end{cases}
$$

which proves the stated result of $\mathcal{I}_{n}^{*} f$.

To derive the compact formula for $\Phi_{n}$ we essentially need a formula for the Dirichlet kernel, denoted by $\Theta_{n}(\mathbf{t})$, of the Fourier series over $\mathbb{H}_{n}^{\dagger *}$,

$$
\Theta_{n}(\mathbf{t})=\sum_{\mathbf{j} \in \mathbb{H}_{n}^{\dagger *}} \phi_{\mathbf{j}}(\mathbf{t})
$$

Indeed, by the definition of $c_{\mathbf{j}}^{(n)}$, it follows that

$$
n^{2} \Phi_{n}(\mathbf{t})=\frac{1}{2}\left(\Theta_{n}(\mathbf{t})+\Theta_{n-1}(\mathbf{t})\right)- \begin{cases}\mathrm{TC}_{\frac{n}{3}, \frac{n}{3},-\frac{2 n}{3}}(\mathbf{t}), & n \equiv 0 \quad(\bmod 3) \\ 0, & \text { otherwise }\end{cases}
$$


Using the identity $\mathbf{k} \cdot \mathbf{t}=\widehat{\mathbf{k}} \cdot \mathbf{s}$ and Proposition 3.5, we derive that

$$
\Theta_{n}(\mathbf{t})=\sum_{\mathbf{k} \in \mathbb{K}_{n}^{\dagger *}} \phi_{\mathbf{k}}(\mathbf{t})=\sum_{\mathbf{k} \in \mathbb{K}_{n}^{\dagger *}} \phi_{\widehat{\mathbf{k}}}(\mathbf{s})=\sum_{\mathbf{j} \in \mathbb{K}_{n}^{*}} \phi_{\mathbf{j}}(\mathbf{s}) .
$$

We now partition $\mathbb{K}_{n}^{*}$ into three parts according to the congruence relation,

$$
\begin{aligned}
& \mathbb{K}_{n}^{(0)}:=\left\{\mathbf{j} \in \mathbb{K}_{n}^{*}: j_{1} \equiv j_{2} \equiv j_{3} \equiv 0 \quad(\bmod 3)\right\}, \\
& \mathbb{K}_{n}^{(1)}:=\left\{\mathbf{j} \in \mathbb{K}_{n}^{*}: j_{1} \equiv j_{2} \equiv j_{3} \equiv 1 \quad(\bmod 3)\right\}, \\
& \mathbb{K}_{n}^{(2)}:=\left\{\mathbf{j} \in \mathbb{K}_{n}^{*}: j_{1} \equiv j_{2} \equiv j_{3} \equiv 2 \quad(\bmod 3)\right\}
\end{aligned}
$$

Using the fact that $\mathbf{j} \in \mathbb{K}_{n}^{(0)} \Longleftrightarrow \frac{\mathbf{j}}{3} \in \mathbb{H}_{\left\lfloor\frac{n}{3}\right\rfloor}^{*}$, where $\mathbb{H}_{n}^{*}$ is the index defined in the previous subsection, and $\phi_{\mathbf{j}}(\mathbf{s})=\phi_{\frac{\mathbf{j}}{3}}(3 \mathbf{s})$ if $\mathbf{j} \in \mathbb{K}_{n}^{(0)}$, we obtain from the Dirichlet kernel over $\mathbb{H}_{n}^{*}$ in (3.10) of [7],

$$
\begin{aligned}
\sum_{\mathbf{j} \in \mathbb{K}_{n}^{(0)}} \phi_{\mathbf{j}}(\mathbf{s}) & =\prod_{j=1}^{3} \frac{\sin \pi\left\lfloor\frac{n+3}{3}\right\rfloor t_{j}}{\sin \pi t_{j}}-\prod_{j=1}^{3} \frac{\sin \pi\left\lfloor\frac{n}{3}\right\rfloor t_{j}}{\sin \pi t_{j}} \\
& =\sum_{j=1}^{3} \frac{\sin 2 \pi\left\lfloor\frac{n}{3}\right\rfloor t_{j}-\sin 2 \pi\left\lfloor\frac{n+3}{3}\right\rfloor t_{j}}{4 \sin \pi t_{1} \sin \pi t_{2} \sin \pi t_{3}}
\end{aligned}
$$

where we have used the identity $\sin 2 t_{1}+\sin 2 t_{2}+\sin 2 t_{3}=-4 \sin t_{1} \sin t_{2} \sin t_{3}$ in the last equal sign [7, (3.15)]. Next we note that $\mathbb{K}_{n}^{(1)}$ can be divided into the following three (non-overlapping) subsets $\mathbb{K}_{n}^{(1)}=\mathbb{K}_{n}^{(1,3)} \cup \mathbb{K}_{n}^{(1,2)} \cup \mathbb{K}_{n}^{(1,1)}$, where

$$
\begin{aligned}
& \mathbb{K}_{n}^{(1,1)}=\left\{\left(-j_{2}-j_{3}, j_{2}, j_{3}\right): j_{2} \equiv j_{3} \equiv 1 \quad(\bmod 3), 1 \leq j_{2},-j_{3} \leq n\right\}, \\
& \mathbb{K}_{n}^{(1,2)}=\left\{\left(j_{1},-j_{1}-j_{3}, j_{3}\right): j_{3} \equiv j_{1} \equiv 1 \quad(\bmod 3), 1 \leq j_{3},-j_{1} \leq n\right\}, \\
& \mathbb{K}_{n}^{(1,3)}=\left\{\left(j_{1}, j_{2},-j_{1}-j_{2}\right): j_{1} \equiv j_{2} \equiv 1 \quad(\bmod 3), 1 \leq j_{1},-j_{2} \leq n\right\} .
\end{aligned}
$$

Using the last set $\mathbb{K}_{n}^{(1,3)}$, we define

$$
\begin{aligned}
& \mathcal{I}\left(t_{1}, t_{2}\right):=\sum_{\mathbf{j} \in \mathbb{K}_{n}^{(1,3)}} \phi_{\mathbf{j}}(\mathbf{s})=\sum_{\substack{1 \leq j_{1} \leq n \\
3 \mid j_{1}-1}} \sum_{\substack{1 \leq-j_{2} \leq n \\
3 \mid j_{2}-1}} \mathrm{e}^{\frac{2 i \pi}{3}\left(j_{2} t_{1}-j_{1} t_{2}\right)} \\
& =\frac{\mathrm{e}^{-\frac{2 i \pi t_{2}}{3}}\left(1-\mathrm{e}^{-2 i \pi\left\lfloor\frac{n+2}{3}\right\rfloor t_{2}}\right)}{1-\mathrm{e}^{-2 i \pi t_{2}}} \frac{\mathrm{e}^{-\frac{4 i \pi t_{1}}{3}}\left(1-\mathrm{e}^{-2 i \pi\left\lfloor\frac{n+1}{3}\right\rfloor t_{1}}\right)}{1-\mathrm{e}^{-2 i \pi t_{1}}} \\
& =\frac{\left(\mathrm{e}^{-2 i \pi s_{2}}-\mathrm{e}^{-2 i \pi s_{1}}\right)\left(1-\mathrm{e}^{-2 i \pi\left\lfloor\frac{n+1}{3}\right\rfloor t_{1}}\right)\left(1-\mathrm{e}^{-2 i \pi\left\lfloor\frac{n+2}{3}\right\rfloor t_{2}}\right)}{\left(1-\mathrm{e}^{-2 i \pi t_{1}}\right)\left(1-\mathrm{e}^{-2 i \pi t_{2}}\right)\left(1-\mathrm{e}^{-2 i \pi t_{3}}\right)},
\end{aligned}
$$

where the second equal sign follows from $\mathbf{j} \cdot \mathbf{s}=j_{1}\left(s_{1}-s_{3}\right)+j_{2}\left(s_{2}-s_{3}\right)=j_{2} t_{1}-j_{1} t_{2}$. Moreover, we have $\mathbf{j} \cdot \mathbf{s}=j_{2} t_{1}-j_{1} t_{2}=j_{3} t_{2}-j_{2} t_{3}=j_{1} t_{3}-j_{3} t_{1}$, which yields

$$
\mathcal{I}\left(t_{2}, t_{3}\right)=\sum_{\mathbf{j} \in \mathbb{K}_{n}^{(1,1)}} \phi_{\mathbf{j}}(\mathbf{s}) \text { and } \mathcal{I}\left(t_{3}, t_{1}\right)=\sum_{\mathbf{j} \in \mathbb{K}_{n}^{(1,2)}} \phi_{\mathbf{j}}(\mathbf{s}) .
$$

As a result, we conclude

$$
\sum_{\mathbf{j} \in \mathbb{K}_{n}^{(1)}} \phi_{\mathbf{j}}(\mathbf{s})=\mathcal{I}\left(t_{1}, t_{2}\right)+\mathcal{I}\left(t_{2}, t_{3}\right)+\mathcal{I}\left(t_{3}, t_{1}\right) .
$$


Furthermore, we note that $\mathbb{K}_{n}^{(2)}=\left\{-\mathbf{j}: \mathbf{j} \in \mathbb{K}_{n}^{(1)}\right\}$ and, consequently,

$$
\begin{aligned}
\sum_{\mathbf{j} \in \mathbb{K}_{n}^{(1)} \cup \mathbb{K}_{n}^{(2)}} \phi_{\mathbf{j}}(\mathbf{s}) & =\sum_{\mathbf{j} \in \mathbb{K}_{n}^{(1)}} \phi_{\mathbf{j}}(\mathbf{s})+\sum_{\mathbf{j} \in \mathbb{K}_{n}^{(2)}} \phi_{\mathbf{j}}(\mathbf{s})=\sum_{\mathbf{j} \in \mathbb{K}_{n}^{(1)}} \phi_{\mathbf{j}}(\mathbf{s})+\sum_{\mathbf{j} \in \mathbb{K}_{n}^{(1)}} \phi_{\mathbf{j}}(-\mathbf{s}) \\
& =\sum_{\mathbf{j} \in \mathbb{K}_{n}^{(1)}} \phi_{\mathbf{j}}(\mathbf{s})+\sum_{\mathbf{j} \in \mathbb{K}_{n}^{(1)}} \overline{\phi_{\mathbf{j}}(\mathbf{s})}=2 \Re\left\{\mathcal{I}\left(t_{1}, t_{2}\right)+\mathcal{I}\left(t_{2}, t_{3}\right)+\mathcal{I}\left(t_{3}, t_{1}\right)\right\} .
\end{aligned}
$$

Now assume that $n$ is a multiple of 3 . By using (3.24) and the fact that $\mathbf{t}$ is homogeneous, we obtain

$$
\begin{aligned}
\sum_{\mathbf{j} \in \mathbb{K}_{n}^{(1)} \cup \mathbb{K}_{n}^{(2)}} \phi_{\mathbf{j}}(\mathbf{s})=2 \Re\left\{\mathcal{I}\left(t_{1}, t_{2}\right)\right)+2 \Re\left(\mathcal{I}\left(t_{2}, t_{3}\right)\right)+2 \Re\left(\mathcal{I}\left(t_{3}, t_{1}\right)\right\} \\
=2 \Re\left\{\frac{\left(\mathrm{e}^{-2 i \pi s_{2}}-\mathrm{e}^{-2 i \pi s_{1}}\right)\left(1+\mathrm{e}^{2 i \pi \frac{n}{3} t_{3}}-\mathrm{e}^{-2 i \pi \frac{n}{3} t_{1}}-\mathrm{e}^{-2 i \pi \frac{n}{3} t_{2}}\right)}{\left(1-\mathrm{e}^{-2 i \pi t_{1}}\right)\left(1-\mathrm{e}^{-2 i \pi t_{2}}\right)\left(1-\mathrm{e}^{-2 i \pi t_{3}}\right)}\right. \\
+\frac{\left(\mathrm{e}^{-2 i \pi s_{3}}-\mathrm{e}^{-2 i \pi s_{2}}\right)\left(1+\mathrm{e}^{2 i \pi \frac{n}{3} t_{1}}-\mathrm{e}^{-2 i \pi \frac{n}{3} t_{2}}-\mathrm{e}^{-2 i \pi \frac{n}{3} t_{3}}\right)}{\left(1-\mathrm{e}^{-2 i \pi t_{1}}\right)\left(1-\mathrm{e}^{-2 i \pi t_{2}}\right)\left(1-\mathrm{e}^{-2 i \pi t_{3}}\right)} \\
\left.+\frac{\left(\mathrm{e}^{-2 i \pi s_{1}}-\mathrm{e}^{-2 i \pi s_{3}}\right)\left(1+\mathrm{e}^{2 i \pi \frac{n}{3} t_{2}}-\mathrm{e}^{-2 i \pi \frac{n}{3} t_{3}}-\mathrm{e}^{-2 i \pi \frac{n}{3} t_{1}}\right)}{\left(1-\mathrm{e}^{-2 i \pi t_{1}}\right)\left(1-\mathrm{e}^{-2 i \pi t_{2}}\right)\left(1-\mathrm{e}^{-2 i \pi t_{3}}\right)}\right\} .
\end{aligned}
$$

Combining the numerators and collecting the terms in $1, \mathrm{e}^{2 i \pi \frac{n}{3}}$ and $\mathrm{e}^{-2 i \pi \frac{n}{3}}$. we obtain that the combined numerator is equal to

$$
\begin{aligned}
& \left(\mathrm{e}^{-2 i \pi s_{2}}-\mathrm{e}^{-2 i \pi s_{1}}\right)+\left(\mathrm{e}^{-2 i \pi s_{3}}-\mathrm{e}^{-2 i \pi s_{2}}\right)+\left(\mathrm{e}^{-2 i \pi s_{1}}-\mathrm{e}^{-2 i \pi s_{3}}\right) \\
& +\left(\mathrm{e}^{-2 i \pi s_{2}}-\mathrm{e}^{-2 i \pi s_{1}}\right) \mathrm{e}^{2 i \pi \frac{n}{3} t_{3}}-\left(\left(\mathrm{e}^{-2 i \pi s_{3}}-\mathrm{e}^{-2 i \pi s_{2}}\right)+\left(\mathrm{e}^{-2 i \pi s_{1}}-\mathrm{e}^{-2 i \pi s_{3}}\right)\right) \mathrm{e}^{-2 i \pi \frac{n}{3} t_{3}} \\
& +\left(\mathrm{e}^{-2 i \pi s_{3}}-\mathrm{e}^{-2 i \pi s_{2}}\right) \mathrm{e}^{2 i \pi \frac{n}{3} t_{1}}-\left(\left(\mathrm{e}^{-2 i \pi s_{1}}-\mathrm{e}^{-2 i \pi s_{3}}\right)+\left(\mathrm{e}^{-2 i \pi s_{2}}-\mathrm{e}^{-2 i \pi s_{1}}\right)\right) \mathrm{e}^{-2 i \pi \frac{n}{3} t_{1}} \\
& +\left(\mathrm{e}^{-2 i \pi s_{1}}-\mathrm{e}^{-2 i \pi s_{3}}\right) \mathrm{e}^{2 i \pi \frac{n}{3} t_{2}}-\left(\left(\mathrm{e}^{-2 i \pi s_{2}}-\mathrm{e}^{-2 i \pi s_{1}}\right)+\left(\mathrm{e}^{-2 i \pi s_{3}}-\mathrm{e}^{-2 i \pi s_{2}}\right)\right) \mathrm{e}^{-2 i \pi \frac{n}{3} t_{2}} \\
= & \left(\mathrm{e}^{-2 i \pi s_{2}}-\mathrm{e}^{-2 i \pi s_{1}}\right)\left(\mathrm{e}^{2 i \pi \frac{n}{3} t_{3}}+\mathrm{e}^{-2 i \pi \frac{n}{3} t_{3}}\right)+\left(\mathrm{e}^{-2 i \pi s_{3}}-\mathrm{e}^{-2 i \pi s_{2}}\right)\left(\mathrm{e}^{2 i \pi \frac{n}{3} t_{1}}+\mathrm{e}^{2 i \pi \frac{n}{3} t_{1}}\right) \\
& +\left(\mathrm{e}^{-2 i \pi s_{1}}-\mathrm{e}^{-2 i \pi s_{3}}\right)\left(\mathrm{e}^{2 i \pi \frac{n}{3} t_{2}}+\mathrm{e}^{-2 i \pi \frac{n}{3} t_{2}}\right) \\
= & 2 \cos \frac{2 \pi n t_{3}}{3} \mathrm{e}^{i \pi s_{3}}\left(\mathrm{e}^{i \pi t_{3}}-\mathrm{e}^{-i \pi t_{3}}\right)+2 \cos \frac{2 \pi n t_{1}}{3} \mathrm{e}^{i \pi s_{1}}\left(\mathrm{e}^{i \pi t_{1}}-\mathrm{e}^{-i \pi t_{1}}\right) \\
& +2 \cos \frac{2 \pi n t_{2}}{3} \mathrm{e}^{i \pi s_{2}}\left(\mathrm{e}^{i \pi t_{2}}-\mathrm{e}^{-i \pi t_{2}}\right) \\
= & 4 i \mathrm{e}^{i \pi s_{3}} \cos \frac{2 \pi n t_{3}}{3} \sin \pi t_{3}+4 i \mathrm{e}^{i \pi s_{1}} \cos \frac{2 \pi n t_{1}}{3} \sin \pi t_{1}+4 i \mathrm{e}^{i \pi s_{2}} \cos \frac{2 \pi n t_{2}}{3} \sin \pi t_{2},
\end{aligned}
$$

where we use the facts that $t_{3}=s_{1}-s_{2}, t_{1}=s_{2}-s_{3}, t_{2}=s_{3}-s_{1}$ and $s_{1}+s_{2}+s_{3}=0$ for the second equal sign. Using $t_{1}+t_{2}+t_{3}=0$, the denominator becomes

$$
\left(1-\mathrm{e}^{-2 i \pi t_{1}}\right)\left(1-\mathrm{e}^{-2 i \pi t_{2}}\right)\left(1-\mathrm{e}^{-2 i \pi t_{3}}\right)=-8 i \sin \pi t_{1} \sin \pi t_{2} \sin \pi t_{3} .
$$

Consequently, we derive that

$$
\begin{aligned}
& \sum_{\mathbf{j} \in \mathbb{K}_{n}^{(1)} \cup \mathbb{K}_{n}^{(2)}} \phi_{\mathbf{j}}(\mathbf{s}) \\
& =2 \Re \frac{\mathrm{e}^{i \pi s_{3}} \cos \frac{2 \pi n t_{3}}{3} \sin \pi t_{3}+\mathrm{e}^{i \pi s_{1}} \cos \frac{2 \pi n t_{1}}{3} \sin \pi t_{1}+\mathrm{e}^{i \pi s_{2}} \cos \frac{2 \pi n t_{2}}{3} \sin \pi t_{2}}{-2 \sin \pi t_{1} \sin \pi t_{2} \sin \pi t_{3}} \\
& \quad=-\sum_{j=1}^{3} \frac{\cos \frac{2 \pi n t_{j}}{3} \cos \pi s_{j} \sin \pi t_{j}}{\sin \pi t_{1} \sin \pi t_{2} \sin \pi t_{3}} .
\end{aligned}
$$


Combining the above equation with (3.22) and (3.23), we obtain

$$
\begin{aligned}
& n^{2} \Phi_{n}(\mathbf{t})=\frac{1}{2}\left(\Theta_{n}(\mathbf{t})+\Theta_{n-1}(\mathbf{t})\right)-\mathrm{TC}_{\frac{n}{3}, \frac{n}{3},-\frac{2 n}{3}}(\mathbf{t}) \\
= & \frac{1}{2}\left(\sum_{\mathbf{j} \in \mathbb{K}_{n}^{(1)}} \phi_{\mathbf{j}}(\mathbf{s})+\sum_{\mathbf{j} \in \mathbb{K}_{n}^{(1)} \cup \mathbb{K}_{n}^{(2)}} \phi_{\mathbf{j}}(\mathbf{s})+\sum_{\mathbf{j} \in \mathbb{K}_{n-3}^{(1)}} \phi_{\mathbf{j}}(\mathbf{s})\right)-\mathrm{TC}_{\frac{n}{3}, \frac{n}{3},-\frac{2 n}{3}}(\mathbf{t}) \\
= & \sum_{j=1}^{3} \frac{\sin \frac{2 \pi(n-3) t_{j}}{3}-\sin \frac{2 \pi(n+3) t_{j}}{3}}{8 \sin \pi t_{1} \sin \pi t_{2} \sin \pi t_{3}}-\sum_{j=1}^{3} \frac{\cos \frac{2 \pi n t_{j}}{3} \cos \pi s_{j} \sin \pi t_{j}}{\sin \pi t_{1} \sin \pi t_{2} \sin \pi t_{3}}-\mathrm{TC}_{\frac{n}{3}, \frac{n}{3},-\frac{2 n}{3}}(\mathbf{t}) \\
= & -\sum_{j=1}^{3} \frac{\cos \frac{2 \pi n t_{j}}{3} \sin \pi t_{j} \cos \pi t_{j}}{2 \sin \pi t_{1} \sin \pi t_{2} \sin \pi t_{3}}-\sum_{j=1}^{3} \frac{\cos \frac{2 \pi n t_{j}}{3} \cos \pi s_{j} \sin \pi t_{j}}{\sin \pi t_{1} \sin \pi t_{2} \sin \pi t_{3}}-\mathrm{TC}_{\frac{n}{3}, \frac{n}{3},-\frac{2 n}{3}}(\mathbf{t}) \\
= & -\sum_{j=1}^{3} \frac{\cos \frac{2 \pi n t_{j}}{3} \sin \pi t_{j}\left(\cos \pi t_{j}+2 \cos \pi s_{j}\right)}{2 \sin \pi t_{1} \sin \pi t_{2} \sin \pi t_{3}}-\frac{1}{3}\left(\cos \frac{2 \pi n t_{1}}{3}+\cos \frac{2 \pi n t_{2}}{3}+\cos \frac{2 \pi n t_{3}}{3}\right) .
\end{aligned}
$$

This completes the proof.

We now proceed to interpolation on the triangle $\Delta$. The idea is to use the periodicity and apply the operator $\mathcal{P}^{ \pm}$in (3.11) on the interpolation $\mathcal{I}_{n} f$, as in Theorem 4.7 in [7. First we apply $\mathcal{P}^{-}$on $\mathcal{I}_{n} f$, which gives the following:

Theorem 3.9. For $n \geq 0$ and $f \in C(\Delta)$ define

$$
\mathcal{L}_{n} f(\mathbf{t}):=\sum_{\mathbf{j} \in \Upsilon_{n}^{\circ}} f\left(\frac{\mathbf{j}}{n}\right) \ell_{\mathbf{j}, n}^{\circ}(\mathbf{t}), \quad \ell_{\mathbf{j}, n}^{\circ}(\mathbf{t})=\frac{6}{n^{2}} \sum_{\mathbf{k} \in \Upsilon_{n}^{\dagger \circ}} \widehat{\lambda}_{\mathbf{k}}^{(n)} \mathrm{TS}_{\mathbf{k}}(\mathbf{t}) \overline{\mathrm{TS}_{\mathbf{k}}\left(\frac{\mathbf{j}}{n}\right)},
$$

where

$$
\widehat{\lambda}_{\mathbf{k}}^{(n)}=c_{\widehat{\mathbf{k}}}^{(3 n)}\left|\mathbf{k} \mathcal{A}_{2}\right|= \begin{cases}6, & k_{1}, k_{2}, n+k_{3}-k_{1}, n+k_{3}-k_{2}>0, \\ 1, & \mathbf{k}=\mathbf{0}, \\ 2, & \mathbf{k}=\left(\frac{n}{3}, \frac{n}{3},-\frac{2 n}{3}\right), \\ \frac{3}{2}, & \mathbf{k}=\left(\frac{n}{2}, 0,-\frac{n}{2}\right) \text { or }\left(0, \frac{n}{2},-\frac{n}{2}\right), \\ 3, & \text { otherwise. }\end{cases}
$$

Then $\mathcal{L}_{n}$ is the unique function in $\mathcal{T} S_{n}$ that satisfies $\mathcal{L}_{n} f\left(\frac{\mathbf{j}}{n}\right)=f\left(\frac{\mathbf{j}}{n}\right), \mathbf{j} \in \Upsilon_{n}^{\circ}$.

Proof. By the definition of $\mathcal{P}^{ \pm}$and $\mathrm{TS}_{\mathbf{k}}$,

$$
\begin{aligned}
\mathcal{P}_{\mathbf{t}}^{-} \Phi\left(\mathbf{t}-\frac{\mathbf{j}}{n}\right) & =\frac{1}{n^{2}} \sum_{\mathbf{k} \in \mathbb{K}_{n}^{\dagger *}} c_{\widehat{\mathbf{k}}}^{(3 n)} \bar{\phi}_{\mathbf{k}}\left(\frac{\mathbf{j}}{n}\right) \mathcal{P}_{\mathbf{t}}^{-} \phi_{\mathbf{k}}(\mathbf{t})=\frac{i}{n^{2}} \sum_{\mathbf{k} \in \mathbb{K}_{n}^{\dagger *}} c_{\widehat{\mathbf{k}}}^{(3 n)} \bar{\phi}_{\mathbf{k}}\left(\frac{\mathbf{j}}{n}\right) \mathrm{TS}_{\mathbf{k}}(\mathbf{t}) \\
& =\frac{i}{n^{2}} \sum_{\mathbf{k} \in \Upsilon_{n}^{\dagger *}} \sum_{\sigma \in \mathcal{A}_{2}} c_{\widehat{\mathbf{k} \sigma}}^{(3 n)} \bar{\phi}_{\mathbf{k} \sigma}\left(\frac{\mathbf{j}}{n}\right) \mathrm{TS} \\
& =\frac{i}{n^{2} \sigma}(\mathbf{t}) \frac{\left|\mathbf{k} \mathcal{A}_{2}\right|}{\left|\mathcal{A}_{2}\right|} \\
& =\frac{1}{n^{2} \in \Upsilon_{n}^{\dagger *}} \sum_{\widehat{\mathbf{k}} \in \Upsilon_{n}^{\dagger *}} c_{\widehat{\mathbf{k}}}^{(3 n)}\left|\mathbf{k} \mathcal{A}_{2}\right| \mathrm{TS} \mathbf{S}_{\mathbf{k}}(\mathbf{t}) \frac{1}{\left|\mathcal{A}_{2}\right|} \sum_{\sigma \in \mathcal{A}_{2}} \operatorname{sign}(\sigma) \bar{\phi}_{\mathbf{k} \sigma}\left(\frac{\mathbf{j}}{n}\right)
\end{aligned}
$$


Now, for $\mathbf{j}, \mathbf{l} \in \Upsilon_{n}^{\circ}$,

$$
\begin{aligned}
& \mathcal{P}_{\mathbf{1}}^{-} \Phi\left(\mathbf{t}-\frac{\mathbf{j}}{n}\right)=\mathcal{P}_{1}^{-} \frac{1}{n^{2}} \sum_{\mathbf{k} \in \mathbb{K}_{n}^{\dagger *}} c_{\widehat{\mathbf{k}}}^{(3 n)} \phi_{\widehat{\mathbf{k}}}\left(\frac{\widehat{\mathbf{l}}}{3 n}\right) \overline{\phi_{\widehat{\mathbf{k}}}\left(\frac{\widehat{\mathbf{j}}}{3 n}\right)}=\mathcal{P}_{\mathbf{1}}^{-} \frac{1}{n^{2}} \sum_{\mathbf{k} \in \mathbb{K}_{n}^{\dagger *}} c_{\widehat{\mathbf{k}}}^{(3 n)} \phi_{\widehat{\widehat{\mathbf{l}}}}\left(\frac{\widehat{\mathbf{k}}}{n}\right) \overline{\phi_{\widehat{\hat{\mathbf{j}}}}\left(\frac{\widehat{\mathbf{k}}}{n}\right)}
\end{aligned}
$$

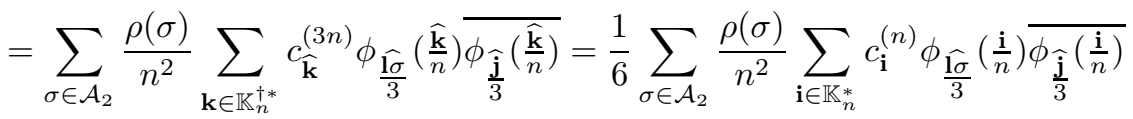

$$
\begin{aligned}
& =\frac{1}{6} \sum_{\sigma \in \mathcal{A}_{2}} \rho(\sigma)\left(\phi_{\frac{\widehat{\sigma} \sigma}{3}}, \phi_{\frac{\widehat{\mathbf{j}}}{3}}\right)_{n}^{*}=\frac{1}{6} \sum_{\sigma \in \mathcal{A}_{2}} \rho(\sigma) \delta_{\mathbf{j}, 1 \sigma}^{n}=\frac{1}{6} \delta_{\mathbf{j}, 1}^{n},
\end{aligned}
$$

where $\delta_{\mathbf{j}, \mathbf{k}}^{n}$ equals 1 if $\frac{\mathbf{j}}{n} \equiv \frac{\mathbf{k}}{n}(\bmod 3)$, and is 0 otherwise. This completes the proof.

In fact, $\ell_{\mathbf{j}, n}^{\circ}(\mathbf{t})=6 \mathcal{P}^{-} \Phi_{n}\left(\mathbf{t}-\frac{\mathbf{j}}{n}\right)$, where $\mathcal{P}^{-}$acts on the variable $\mathbf{t}$, from which the proof reduces to verify formula of $\ell_{\mathbf{j}}^{\circ}$ given in the theorem, using the periodicity and the symmetry. Applying now $\mathcal{P}^{+}$to $\mathcal{I}_{n} f$, we obtain similarly the trigonometric interpolation on $\Upsilon_{n}$ in $\Delta$.

Theorem 3.10. For $n \geq 0$ and $f \in C(\Delta)$ define

$$
\mathcal{L}_{n}^{*} f(\mathbf{t}):=\sum_{\mathbf{j} \in \Upsilon_{n}} f\left(\frac{\mathbf{j}}{n}\right) \ell_{\mathbf{j}, n}(\mathbf{t}), \quad \ell_{\mathbf{j}, n}(\mathbf{t}):=\frac{\lambda_{\mathbf{j}}^{(n)}}{n^{2}} \sum_{\mathbf{k} \in \Upsilon_{n}^{\dagger}} \lambda_{\mathbf{k}}^{(n)} \mathrm{TC}_{\mathbf{k}}(\mathbf{t}) \overline{\mathrm{TC}}\left(\frac{\mathbf{j}}{n}\right),
$$

where $\lambda_{\mathbf{j}}^{(n)}$ are defined in (3.19). Then $\mathcal{L}_{n}^{*}$ is the unique function in $\mathcal{T} C_{n}$ that satisfies $\mathcal{L}_{n}^{*} f\left(\frac{\mathbf{j}}{n}\right)=f\left(\frac{\mathbf{j}}{n}\right), \mathbf{j} \in \Upsilon_{n}$.

For $n$ being a multiple of 3 , we can deduce a compact formula for $\ell_{\mathbf{j}, n}^{\circ}(\mathbf{t})$ and $\ell_{\mathbf{j}, n}(\mathbf{t})$ from that of (3.20). The interpolation points of $\mathcal{L}_{n}^{*} f$ are depicted in Figure 8. From the explicit formula of $\Phi_{n}^{*}$ in (3.20), it is not difficult to prove, following proof of Theorem 3.6 in [7, that the uniform operator norm (Lebesgue constant) of $\mathcal{I}_{n}^{*} f$ in Theorem 3.8 satisfies $\left\|\mathcal{I}_{n}^{*}\right\|_{\infty} \leq c(\log n)^{2}$ for $n \equiv 0(\bmod 3)$; in other words, $\left\|\mathcal{I}_{n}^{*} f\right\|_{\infty} \leq c\|f\|_{\infty}$, where $\|\cdot\|_{\infty}$ denotes the uniform norm over $\Omega$. Since $\mathcal{L}_{n} f$ and $\mathcal{L}_{n}^{*} f$ are obtained by applying $\mathcal{P}^{ \pm}$to $\mathcal{I}_{n}^{*} f$, it follows immediately that

$$
\left\|\mathcal{L}_{n}\right\|_{\infty} \leq c(\log n)^{2} \quad \text { and } \quad\left\|\mathcal{L}_{n}^{*}\right\|_{\infty} \leq c(\log n)^{2},
$$

where $n \equiv 0(\bmod 3)$ and the uniform norm is taken over the triangle $\Delta$.

3.5.3. Fast Fourier transform. Comparing to the Hexagon-Hexagon case, the set up in the present subsection has at least one advantage if we consider the fast Fourier transform. The discrete Fourier transform of a function $f$ periodic in $H$ is

$$
\mathcal{I}_{n} f(\mathbf{t})=\sum_{\mathbf{k} \in \mathbb{K}_{n}^{\dagger}} \widehat{f}_{\mathbf{k}} \phi_{\mathbf{k}}(\mathbf{t}), \quad \text { where } \quad \widehat{f}_{\mathbf{k}}=\left\langle f, \phi_{\mathbf{k}}\right\rangle_{n}=\frac{1}{n^{2}} \sum_{\mathbf{j} \in \mathbb{K}_{n}} f\left(\frac{\mathbf{j}}{n}\right) \mathrm{e}^{-\frac{2 i \pi}{3} \mathbf{k} \cdot \mathbf{j}} .
$$

For $\langle\cdot, \cdot\rangle_{n}$ in (3.17), we show that $\widehat{f}_{\mathbf{k}}$ can be evaluated as in the classical discrete Fourier transform on a square. For this purpose, it is more convenient to use Cartesian coordinates. Let $k=\left(k_{1}, k_{2}\right)$ corresponds to $\mathbf{k}$. Then, by Theorem 2.2 ,

$$
\widehat{f}_{\mathbf{k}}=\left(f, \phi_{\mathbf{k}}\right)_{n}=\left\langle f, \phi_{k}\right\rangle_{N}=\frac{1}{n^{2}} \sum_{j \in \Lambda_{N}} f\left(n^{-1} H j\right) \mathrm{e}^{2 i \pi n^{-1} k \cdot j},
$$


since $\phi_{k}(x)=\mathrm{e}^{2 \pi i H^{-1} k \cdot x}$ and $B=n H^{-\operatorname{tr}}$ implies that $\phi_{k}\left(B^{-\operatorname{tr}} j\right)=\mathrm{e}^{2 i \pi n^{-1} k \cdot j}$. The homogeneous coordinates of $H j$ is $\left(2 j_{1}-j_{2}, 2 j_{2}-j_{1},-j_{1}-j_{2}\right)$, so that

$$
\begin{aligned}
\hat{f}_{\mathbf{k}} & =\frac{1}{n^{2}} \sum_{j \in \Lambda_{N}} f\left(\frac{2 j_{1}-j_{2}}{n}, \frac{2 j_{2}-j_{1}}{n}, \frac{-j_{1}-j_{2}}{n}\right) \mathrm{e}^{2 i \pi n^{-1} k \cdot j} \\
& =\frac{1}{n^{2}} \sum_{0 \leq j_{1}, j_{2}<n} f\left(\frac{2 j_{1}-j_{2}}{n}, \frac{2 j_{2}-j_{1}}{n}, \frac{-j_{1}-j_{2}}{n}\right) \mathrm{e}^{2 i \pi n^{-1}\left(k_{1} j_{1}+k_{2} j_{2}\right)} .
\end{aligned}
$$

This states that the discrete Fourier transform coincides, up to a reordering, with the classical discrete Fourier transform on a rectangle. Figure 9 shows the set $\Lambda_{N}$ and its reordering in rectangular coordinates. Similarly, recalling $\mathcal{H}_{N}=\left\{\phi_{\mathbf{k}}: \mathbf{k} \in\right.$
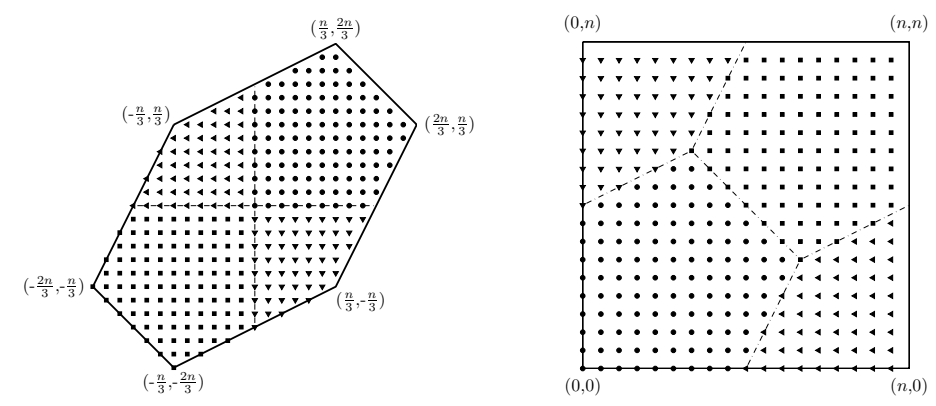

Figure 9. The index set $\Lambda_{N}$ (left) and its reordering (right).

$\left.\mathbb{K}_{n}^{\dagger}\right\}$; the index set $\mathbb{K}_{n}^{\dagger}$ in rectangular coordinates can also be reordered, so that $\mathcal{H}_{N}$ becomes the product space in rectangular domain. In particular, this allows us to apply the classical FFT to evaluate $\widehat{\hat{f}_{\mathbf{k}}}$.

3.6. Other possibilities. There are other possible choices of lattices in our general frame of discrete Fourier analysis. For example, we can consider $A=H^{- \text {tr }}$ and $B=n H$, for which the integral domain $\Omega_{A}$ will be the hexagon in Figure 5 . It is easy to see that the index sets $\Lambda_{N}$ and $\Lambda_{N}^{\dagger}$ in this case are $\mathbb{K}_{n}^{\dagger}$ and $\mathbb{K}_{n}$ in the previous subsection, that is, their roles are interchanged. This case, however, does not seem to lead to interesting new result; the integral domain in the Stage 3 for the generalized cosine and sine functions will be the quadrilateral in Figure 7 .

One obvious question is if we can choose one lattice tiling $\mathbb{R}^{2}$ with square or rhombus and choose the other lattice tiling $\mathbb{R}^{2}$ with hexagon. The answer is negative if we try to use regular hexagon, since the matrix $H$ contains $\sqrt{3}$ and the requirement $N=B^{\operatorname{tr}} A$ having all integer entries cannot be satisfied. We can, however, use other hexagon domains. For example, we can choose either

$$
H_{1}=\left[\begin{array}{cc}
1 & 1 \\
-2 & 1
\end{array}\right] \quad \text { or } \quad H_{2}=\left[\begin{array}{cc}
1 & 2 \\
-1 & 1
\end{array}\right]
$$

Both lattices $H_{1} \mathbb{Z}^{2}$ and $H_{2} \mathbb{Z}^{2}$ tile $\mathbb{R}^{2}$. Their fundamental domains are depicted in Figure 10. The general result in Section 2.1 can be applied to develop a discrete Fourier analysis using either $H_{1}$ or $H_{2}$ and a lattice that tiles $\mathbb{R}^{2}$ with either square or rhombus, since the requirement that $N=B^{\operatorname{tr}} A$ has integer entries can be readily attained using, say $A=H_{1}$ or $H_{2}$ and $B=I$ or $R$. Comparing to the regular hexagon, the hexagons in Figure 9 possess far less symmetry. The lack of symmetry 

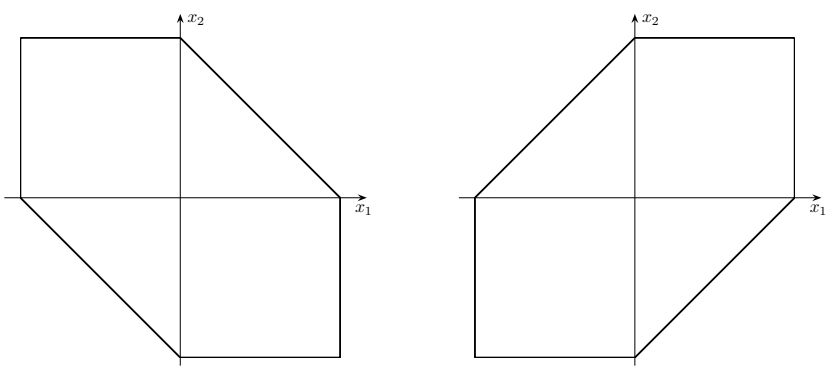

Figure 10. The fundamental domains of $H_{1} \mathbb{Z}^{2}$ (left) and $H_{2} \mathbb{Z}^{2}$ (right).

means that we will not be able to carry the program outlined in Section 2.2 to Stage 3 and Stage 4, whereas the results in Stage 1 and Stage 2 can be derived from the general theory straightforwardly. Hence, we will not pursuit the matter any further.

\section{REFERENCES}

[1] J. H. Conway and N. J. A. Sloane, Sphere Packings, Lattices and Groups, 3rd ed. Springer, New York, 1999.

[2] D. E. Dudgeon and R. M. Mersereau, Multidimensional Digital Signal Processing, PrenticeHall Inc, Englewood Cliffs, New Jersey, 1984.

[3] C. F. Dunkl and Yuan Xu, Orthogonal polynomials of several variables, Encyclopedia of Mathematics and its Applications, vol. 81, Cambridge Univ. Press, 2001.

[4] B. Fuglede, Commuting self-adjoint partial differential operators and a group theoretic problem, J. Functional Anal. 16 (1974), 101-121.

[5] J. R. Higgins, Sampling theory in Fourier and Signal Analysis, Foundations, Oxford Science Publications, New York, 1996.

[6] T. Koornwinder, Orthogonal polynomials in two varaibles which are eigenfunctions of two algebraically independent partial differential operators, Nederl. Acad. Wetensch. Proc. Ser. A77 = Indag. Math. 36 (1974), 357-381.

[7] H. Li, J. Sun and Y. Xu, Discrete Fourier analysis, cubature and interpolation on a hexagon and a triangle, SIAM J. Numer. Anal., 46 (2008) 1653-1681.

[8] H. Li, J. Sun and Y. Xu, Cubature formula and interpolation on the cubic domain. Numer Math: Theory, Method and Appl. 2 (2009), 119-152.

[9] H. Li and Y. Xu, Discrete Fourier analysis on a dodecahedron and a tetrahedron, Math. Comp. 78 (2009) 999-1029.

[10] H. Li and Y. Xu, Discrete Fourier analysis on fundamental domain and simplex of $A_{d}$ lattice in $d$-variables, J. Fourier Anal. Appl., Online First DOI 10.1007/s00041-009-9106-9.

[11] De Marchi, M. Vianello and Y. Xu, New cubature formulae and hyperinterpolation in three variables, BIT Numer. Math., 49 (2009), 55-73.

[12] H. Möller, Kubaturformeln mit minimaler Knotenzahl, Numer. Math. 25 (1976), p. 185-200.

[13] C. R. Morrow and T. N. L. Patterson, Construction of algebraic cubature rules using polynomial ideal theory, SIAM J. Numer. Anal., 15 (1978), 953-976.

[14] R. J. Marks II, Introduction to Shannon Sampling and Interpolation Theory, Springer-Verlag, New York, 1991.

[15] H. Z. Munthe-Kaas, On group Fourier analysis and symmetry preserving discretizations of PDEs, J. Phys. A: Math. Gen., 39 (2006), 5563-5584.

[16] I. P. Mysoviskikh, Interpolatory cubature formulas, Nauka, Moscow, 1981.

[17] H. J. Schmid and Y. Xu, On bivariable Gaussian cubature formulae, Proc. Amer. Math. Soc. 122 (1994), 833-842.

[18] A. Stroud, Approximate calculation of multiple integrals, Prentice-Hall, Englewood Cliffs, NJ, 1971.

[19] J. Sun, Multivariate Fourier series over a class of non tensor-product partition domains, J. Comput. Math. 21 (2003), 53-62. 
[20] Y. Xu, Common Zeros of Polynomials in Several Variables and Higher Dimensional Quadrature, Pitman Research Notes in Mathematics Series, Longman, Essex, 1994.

[21] Y. Xu, Lagrange interpolation on Chebyshev points of two variables, J. Approx. Theory, 87 (1996), 220-238.

[22] Y. Xu, Fourier series and approximation on hexagonal and triangular domains. Constructive Approx., to appear.

[23] A. Zygmund, Trigonometric series, Cambridge Univ. Press, Cambridge, 1959.

Institute of Software, Chinese Academy of Sciences, Beijing 100190, China

E-mail address: hynli@mail.rdcps.ac.cn

Institute of Software, Chinese Academy of Sciences, Beijing 100190, China

E-mail address: sun@mail.rdcps.ac.cn

Department of Mathematics, University of Oregon, Eugene, Oregon 97403-1222.

E-mail address: yuan@math.uoregon.edu 